\title{
Trajectory of the turning point is dense for a co- $\sigma$-porous set of tent maps
}

\author{
by \\ Karen B r u cks (Milwaukee, WI) and \\ Zoltán Buczolich (Budapest)
}

\begin{abstract}
It is known that for almost every (with respect to Lebesgue measure) $a \in[\sqrt{2}, 2]$ the forward trajectory of the turning point of the tent map $T_{a}$ with slope $a$ is dense in the interval of transitivity of $T_{a}$. We prove that the complement of this set of parameters of full measure is $\sigma$-porous.
\end{abstract}

1. Introduction. For $a \in(1,2]$ set $T_{a}(x)=a x$ for $0 \leq x \leq 1 / 2$ and $T_{a}(x)=a(1-x)$ for $1 / 2 \leq x \leq 1$. We refer to this family of maps as the family of tent maps. Other models are possible, but two tent maps with the same slope are conjugate via an affine transformation and hence the model does not matter. We choose this model as it makes our computations the easiest. The only measure we use is Lebesgue measure.

We restrict our attention to the parameters $a$ from $[\sqrt{2}, 2]$. If $\sqrt{2}<a^{m}$ $\leq 2$ for some $m \in\left\{1,2,2^{2}, 2^{3}, \ldots\right\}$, then the nonwandering set of $T_{a}$ consists of $m$ disjoint closed intervals and a finite number of periodic points [15, p. 78]. Moreover, for such $a$ the map $T_{a}^{m}$ restricted to any one of those intervals is a tent map with slope $a^{m}$, so is affinely conjugate to $T_{a^{m}}$. Thus, getting corresponding results for smaller parameter values is easy. We work with $T_{a}$ restricted to its core, $\left[T_{a}^{2}(1 / 2), T_{a}(1 / 2)\right]$; the core is the smallest forward invariant interval containing the turning point $1 / 2$. In fact, $T_{a}$ is transitive on the core. For more information on transitivity, nonwandering sets, and other related topics see [1]. The term trajectory will always refer to the forward trajectory.

2000 Mathematics Subject Classification: Primary 58F03; Secondary 54H20, 26A21.

Research of K. Brucks supported in part by a Fulbright grant while visiting the Eötvös Loránd University, Budapest.

Z. Buczolich was supported by the Hungarian National Foundation of Scientific Research Grant No. 019476 and FKFP 0189/1997. 
In [3] it was proven that for almost every (with respect to Lebesgue measure) $a \in[\sqrt{2}, 2]$, the $T_{a}$ trajectory of the turning point $1 / 2$ is dense in $\left[T_{a}^{2}(1 / 2), T_{a}(1 / 2)\right]$. Letting $\mathcal{D}$ denote those parameters $a \in[\sqrt{2}, 2]$ such that the closure of the trajectory of $1 / 2$ under $T_{a}$ is $\left[T_{a}^{2}(1 / 2), T_{a}(1 / 2)\right]$, we prove:

THEOREM 1. The set $[\sqrt{2}, 2] \backslash \mathcal{D}$ is $\sigma$-porous.

In Section 2 we give basic definitions related to porosity and $\sigma$-porosity. For a detailed survey of these concepts we refer to [17] and the appendix of [16]. Each $\sigma$-porous set in $\mathbb{R}$ is of the first category and of zero Lebesgue measure. These sets arise quite often as exceptional sets. For example, Preiss and Zajíček verified that the set of points of Fréchet nondifferentiability of any continuous convex function on a Banach space with a separable dual is $\sigma$-porous [12]. However, Konyagin showed that the set $E=\{x \in \mathbb{R} \mid$ $\left.\sum_{n=1}^{\infty}|\sin (n ! \pi x) / n| \leq 1\right\}$ is a closed non- $\sigma$-porous set of zero Lebesgue measure [17, Chapter 5]. This shows that the $\sigma$-ideal of $\sigma$-porous sets is a proper subset of the $\sigma$-ideal of measure zero first category sets. Therefore Theorem 1 strengthens the result in [3]. To obtain this stronger result, a more delicate (refined) study of the kneading properties of tent maps was necessary. Some of these techniques might be of independent interest.

We break the proof of Theorem 1 into two cases. In Section 4 we deal with the easier case, namely, parameters $a$ such that $\liminf _{k \rightarrow \infty} Q_{a}(k)<\infty$, where $Q_{a}(k)$ denotes the kneading map of $T_{a}$. The remainder of the paper deals with parameters $a$ such that $\lim _{k \rightarrow \infty} Q_{a}(k)=\infty$. Kneading maps and Hofbauer towers are recalled in the next section.

When obtaining measure results for one-parameter families of unimodal maps, one often deals with the piecewise monotone functions $\xi_{n}(a) \equiv f_{a}^{n}(c)$, where $\left\{f_{a}\right\}$ is the one-parameter family of maps with common turning point $c$ and $n \in \mathbb{N}$. Given an $n$, the laps of $\xi_{n}(a)$ are the maximal subintervals of monotonicity of $\xi_{n}$. In [3], where $f_{a}=T_{a}$, the main tool is the following: there exists $\varepsilon>0$ such that for almost every $a \in[\sqrt{2}, 2]$ and for every $M \in \mathbb{N}$, there is an $n \geq M$ such that $c \in \xi_{n}(J)$ and $\left|\xi_{n}(J)\right|>\varepsilon$ for the lap $J$ of $\xi_{n}$ containing $a$; here the measure of $\xi_{n}(J)$ is denoted by $\left|\xi_{n}(J)\right|$. In less precise terms, one produces long stretches in the graphs of the $\xi_{n}$ 's for almost all $a$ and for arbitrarily large $n$. Similar such long stretches have been used in obtaining other measure results $[4,8,14]$. For our porosity results we need to produce long stretches with some additional measure properties/estimates on the associated laps in parameter space; this is done in Section 6 .

For a given tent map, $T_{a}$, the levels of the Hofbauer tower will be denoted by $D_{n}(a)$. As remarked above, we first treat the easier case, namely when $\liminf _{k \rightarrow \infty} Q_{a}(k)<\infty$, or equivalently (see Lemma 1), $\liminf _{n \rightarrow \infty}\left|D_{n}(a)\right|$ $>0$. In this case, infinitely many of the levels in the Hofbauer tower contain a "long stretch" (denoted by $W$ in Section 4 ). This long stretch is 
used to get the porosity result. Since $\lim _{k \rightarrow \infty} Q_{a}(k)=\infty$ is equivalent to $\lim _{n \rightarrow \infty}\left|D_{n}(a)\right|=0$, it is more difficult to find the required long stretches in this second case. To understand the dynamics behind the formal proof presented in Section 6 we provide an algorithm, named the substantial cut algorithm, which finds the required long stretches suitable for the porosity estimates. This is done in Section 5. A second algorithm, called the greedy algorithm, is also discussed. This algorithm provides long stretches in a "fast" way, but these stretches are not suitable for the geometric estimates needed for porosity. However, the greedy algorithm can be used to obtain lower estimates of the length of levels of the Hofbauer tower at certain cutting times which we call substantial cuts (see Lemma 9).

2. Preliminaries. Let $(X, \varrho)$ be a compact metric space, $E \subset X, x \in$ $X$, and $\delta>0$. Then $E^{\mathrm{c}}=X \backslash E$ and $B(x, \delta)=\{y \in X \mid \varrho(x, y)<\delta\}$. We define $\gamma(E, x, \delta)$ to be the minimum of 1 and the number defined by

$$
\sup \left\{2 \eta \mid \eta>0 \text { and there exists } y \in X \text { such that } B(y, \eta) \subset B(x, \delta) \cap E^{\mathrm{c}}\right\} .
$$

If no such $y$ exists, we set $\gamma(E, x, \delta)=0$. We can now define the porosity of $E$ in $X$. For more detailed discussions on porosity see $[13,16,17]$. be

Definition 1. If $x \in E$, then we define the porosity of $E$ in $X$ at $x$ to

$$
p(E, x)=\limsup _{\delta \rightarrow 0^{+}} \frac{\gamma(E, x, \delta)}{\delta} .
$$

If $p(E, x)>0$, then $E$ is said to be porous in $X$ at $x$. We say that $E$ is porous in $X$ if $p(E, x)>0$ for all $x \in E$. Any subset of $X$ which can be written as a countable union of sets, each porous in $X$, is said to be $\sigma$-porous in $X$. If $A \subset X$ is $\sigma$-porous, then we say $X \backslash A$ is co- $\sigma$-porous.

Notice that if $X$ contains no isolated points, then any countable subset of $X$ is $\sigma$-porous. For $E, F \subset X$, we denote the Hausdorff distance between $E$ and $F$ by $\operatorname{HD}(E, F)$; so $\operatorname{HD}(E, F)=\max \left\{d_{E}(F), d_{F}(E)\right\}$, where $d_{A}(B)=$ $\sup \{\varrho(A, x) \mid x \in B\}$. We denote the closure of a set $U \subset X$ by $\bar{U}$.

A continuous map $f:[0,1] \rightarrow[0,1]$ is called unimodal if there exists a unique turning or critical point, $c$, such that $\left.f\right|_{[0, c)}$ is increasing, $\left.f\right|_{(c, 1]}$ is decreasing, and $f(0)=f(1)=0$. To avoid trivial cases, we assume that $f(c)>c>f(f(c))$. We denote the forward images of $c$ by $c_{i}=f^{i}(c)$. Clearly the interval $\left[c_{2}, c_{1}\right]$ is invariant and $f$ maps $\left[c_{2}, c_{1}\right]$ onto itself; the interval $\left[c_{2}, c_{1}\right]$ is called the core of the map $f$.

Let $f^{n}$ be some iterate of $f$ and let $J$ be any maximal subinterval on which $f^{n} \mid J$ is monotone. Then $f^{n}: J \rightarrow[0,1]$ is called a branch of $f^{n}$. A branch $f^{n}: J \rightarrow[0,1]$ is called a central branch if $c \in \partial J$. Hence there are always two central branches, and their images are the same. An iterate $n$ 
is called a cutting time if the image of the central branch of $f^{n}$ contains $c$. The cutting times are denoted by $S_{0}, S_{1}, S_{2}, \ldots\left(S_{0}=1\right.$ and $\left.S_{1}=2\right)$. If $f^{S_{k}}: J \rightarrow[0,1]$ is the left central branch of $f^{S_{k}}$, then there is a unique point $z_{k} \in J$ such that

$$
f^{S_{k}}\left(z_{k}\right)=c .
$$

By construction, $z_{k}$ has the property that $\bigcup_{0<j \leq S_{k}} f^{-j}(c) \cap\left(z_{k}, c\right)=\emptyset$ and is therefore called a closest precritical point. The point $\widehat{z}_{k}$, defined analogously for the right central branch of $f^{S_{k}}$, is also a closest precritical point. It can be proven that the difference of two consecutive cutting times is again a cutting time. Hence we can write

$$
S_{k}-S_{k-1}=S_{Q(k)},
$$

where $Q: \mathbb{N} \rightarrow \mathbb{N}$ is an integer function, called the kneading map. An equivalent statement is

$$
c_{S_{k}} \in\left(z_{Q(k+1)-1}, z_{Q(k+1)}\right] \cup\left[\widehat{z}_{Q(k+1)}, \widehat{z}_{Q(k+1)-1}\right) .
$$

The kneading map was introduced by Hofbauer (see e.g. $[9,10]$ ). If $Q(k)$ is defined for all $k \in \mathbb{N}$, then

$$
Q(k)<k
$$

for all $k \in \mathbb{N}$; one can easily see that this follows from (2), cf. also [5, page 1341]. The kneading map (or cutting times) determines the combinatorics of $f$ completely. A survey of this tool can be found in [5]; our discussion follows [5].

Closely related to the kneading map is the Hofbauer tower [9]. Given a unimodal map $f$, the associated Hofbauer tower is the disjoint union of intervals $\left\{D_{n}\right\}_{n \geq 1}$, where $D_{1}=\left[0, c_{1}\right]$ and, for $n \geq 2$,

$$
D_{n+1}= \begin{cases}f\left(D_{n}\right) & \text { if } c \notin D_{n}, \\ {\left[c_{n+1}, c_{1}\right]} & \text { if } c \in D_{n} .\end{cases}
$$

Notice that the image of either central branch $f^{n}: J \rightarrow[0,1]$ is such that $f^{n}(J)=D_{n}$. From (2) it follows that for $k \geq 1$,

$$
D_{S_{k}}=\left[c_{S_{k}}, c_{S_{Q(k)}}\right] \text {. }
$$

We say a unimodal map $f$ is locally eventually onto (leo) provided that for every $\varepsilon>0$ there exists $M \in \mathbb{N}$ such that if $U$ is an interval with $|U|>\varepsilon$ and if $n \geq M$, then $f^{n}(U)=\left[c_{2}, c_{1}\right]$. We say $x \in I$ is periodic provided there exists $n \in \mathbb{N}$ such that $f^{n}(x)=x$. Similarly, we say $x \in I$ is eventually periodic provided there exists $n \in \mathbb{N}$ and a periodic point $y$ such that $f^{n}(x)=y$. When writing $[a, b]$ we do not assume that $a \leq b$. We denote the rationals by $\mathbb{Q}$ and the length of an interval $U$ by $|U|$.

3. Tent map preliminaries. For each $a \in[\sqrt{2}, 2]$, the map $T_{a} \mid\left[c_{2}, c_{1}\right]$ is leo; see e.g. $[2$, Lemma 2]. Let $\mathcal{P}=\{a \in[\sqrt{2}, 2] \mid$ the turning point 
$1 / 2$ is either periodic or eventually periodic $\}$. The set $\mathcal{P}$ is countable and contains no isolated points [2]; therefore $\mathcal{P}$ is $\sigma$-porous. Also, for $a \in \mathcal{P}$, the kneading map $Q_{a}(k)$ is defined for only finitely many $k \in \mathbb{N}$ (see e.g. [5, page 1341]). Next, notice that for any $a \in(1,2]$ the $z_{k}$ 's as defined in (1) are such that $\widehat{z}_{k}=1-z_{k}$ and $\lim _{k \rightarrow \infty} z_{k}=\lim _{k \rightarrow \infty} \widehat{z}_{k}=1 / 2$. When more than one-parameter value is being used, we may write $D_{n}(a)$ for the levels in the Hofbauer tower for $T_{a}, Q_{a}(k)$ for the kneading map of $T_{a}, S_{k}(a)$ for the cutting times of $T_{a}$, or $c_{n}(a)$ for $T_{a}^{n}(c)$. Another notation for $c_{n}(a)$ is $\xi_{n}(a)$, the latter being used when one is interested in $T_{a}^{n}(c)$ as a function of the parameter $a$.

\section{DeFinition 2. Set}

$$
\begin{aligned}
& \mathcal{D}=\left\{a \in[\sqrt{2}, 2] \mid{\overline{\left\{T_{a}^{n}(c)\right\}_{n \geq 0}}}_{\left.n=\left[c_{2}(a), c_{1}(a)\right]\right\},}\right. \\
& \mathcal{I}=\left\{a \in[\sqrt{2}, 2] \mid \lim _{k \rightarrow \infty} Q_{a}(k)=\infty\right\} .
\end{aligned}
$$

As noted in the introduction, it is easy to establish that $\{a \in[\sqrt{2}, 2] \mid$ $\liminf _{k \rightarrow \infty} Q_{a}(k)<\infty$ and $\left.a \notin \mathcal{D} \cup \mathcal{P}\right\}$ is $\sigma$-porous; this is done in Section 4 . The more interesting/difficult case is to show that $\mathcal{I}$ is $\sigma$-porous; this is done in Sections 6 and 7.

It is known that for $a \in \mathcal{D}$ we have $\liminf _{k \rightarrow \infty} Q_{a}(k)<2$; see e.g. $[4$, Lemma 3.5] (this is not an "if and only if" statement). Hence, $\mathcal{D} \cap \mathcal{I}=\emptyset$. Again, in [3] it is shown that $\mathcal{D}$ has full Lebesgue measure in $[\sqrt{2}, 2]$ and hence $\mathcal{I}$ has zero Lebesgue measure in $[\sqrt{2}, 2]$. On the other hand, $\mathcal{I}$ is dense in $[\sqrt{2}, 2]$ and is uncountable. Since we could not find a proof of this fact in the literature, we include it for completeness (Lemma 5). We do not explicitly use Lemmas 1 and 5 in the paper, but we include them to give/recall facts about the set $\mathcal{I}$.

Lemma 1. Fix $a>\sqrt{2}$. Let $Q(k)$ be the kneading map for $T_{a}$. Then $\lim _{k \rightarrow \infty} Q(k)=\infty$ if and only if $\lim _{n \rightarrow \infty}\left|D_{n}\right|=0$.

Proof. If $\lim _{n \rightarrow \infty}\left|D_{n}\right|=0$, then (3) and (5) imply $\lim _{k \rightarrow \infty} Q(k)=\infty$.

If $\lim _{k \rightarrow \infty} Q(k)=\infty$, then (3) and (5) imply $\lim _{k \rightarrow \infty}\left|D_{S_{k}}\right|=0$. But $\left|D_{n}\right|<\left|D_{S_{k+1}}\right|$ for $S_{k}<n<S_{k+1}$ since $T_{a} \mid D_{n}$ is monotone for such $n$. Thus, $\lim _{n \rightarrow \infty}\left|D_{n}\right|=0$.

Lemma 1 holds for more general unimodal maps with some expansion properties.

Definition 3. For $a \in[\sqrt{2}, 2]$ and $n \in \mathbb{N}$ let $\omega_{n}(a)$ be the maximal open interval in the parameter space containing $a$ such that $\xi_{n}$ is monotone on $\omega_{n}(a)$; recall that $\xi_{n}(a) \equiv T_{a}^{n}(c)$. Note that $\omega_{n}(a)$ is not defined for $a \in \mathcal{P}$ and large $n$. In Figure 1, with $n=S_{k}$, we have $\omega_{S_{k}}(a)=(u, v)$. 


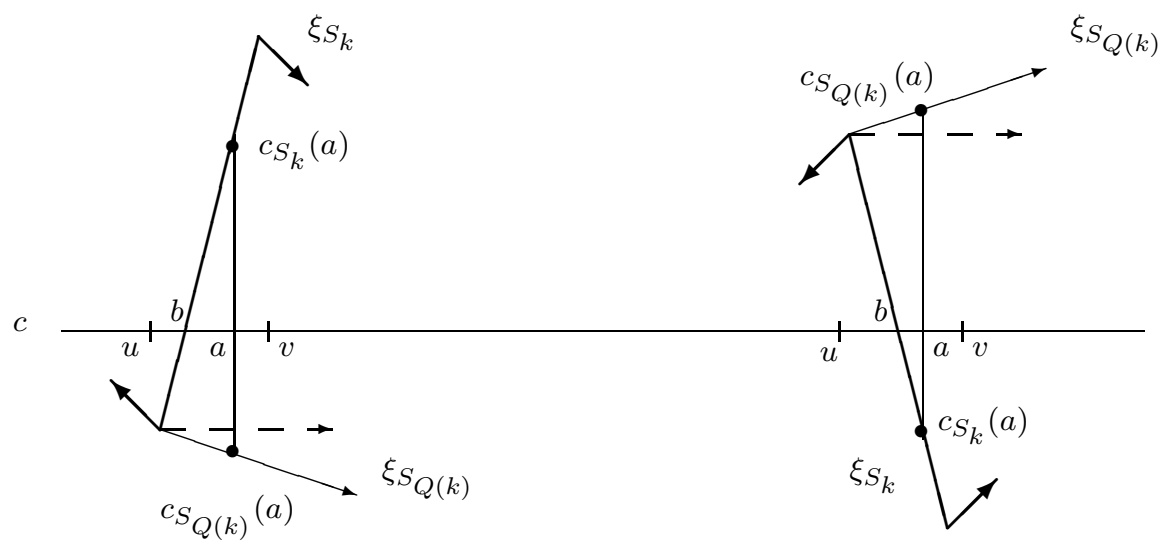

Fig. 1. Phase Space at cutting time $S_{k}$

We recall two known lemmas (see e.g. [3, 14]).

Lemma 2. Fix $\varepsilon_{0}>0$ and $a_{0} \in(\sqrt{2}, 2]$. Then there exists $K_{0} \in \mathbb{N}$ such that for all $k \geq K_{0}$,

$$
\frac{\left|\xi_{k}^{\prime}(b)\right|}{\left|\xi_{k}^{\prime}(a)\right|} \leq 1+\varepsilon_{0}
$$

whenever $a, b$, belong to the same lap of $\xi_{n} \mid\left[a_{0}, 2\right]$.

Lemma 3. There exist positive constants $\alpha$ and $\beta$ such that for all $k \geq 2$ and all $a \in[\sqrt{2}, 2]$,

$$
\alpha a^{k} \leq\left|\xi_{k}^{\prime}(a)\right| \leq \beta a^{k}
$$

wherever $\xi_{k}^{\prime}$ is defined. Hence we also have

$$
\frac{1}{\alpha} a^{-k} \geq\left|\left(\xi_{k}^{-1}\right)^{\prime}\left(\xi_{k}(a)\right)\right| \geq \frac{1}{\beta} a^{-k}
$$

for any branch of $\xi_{k}^{-1}$.

For a discussion of Lemma 4 see [14, Chapter 3]. Lemma 4 is used only in the proof of Lemma 5.

Lemma 4. Fix $a \in(\sqrt{2}, 2]$. Then $n$ is a cutting time for $T_{a}$ if and only if $\xi_{n}\left(\omega_{n}(a)\right) \ni c$ and $a>b$ where $b$ is the unique point in $\omega_{n}(a)$ such that $\xi_{n}(b)=c$.

One often works in Phase Space, i.e., one plots the $\xi_{n}(a)$ 's as functions of the parameter $a$. Figure 1 is a piece of Phase Space. Let $a$ be given and suppose that $n=S_{k}(a)=S_{k}$ is a cutting time for $T_{a}$; let $Q_{a}(k)=$ $Q(k)$. Then one of the pictures in Figure 1 holds. From Lemma 2, we see that for large $n$ the graph of $\xi_{n}$ is almost linear and hence for ease we draw linear functions in Figure 1; thus assume that $k$ is large in Figure 1. We have $\omega_{S_{k}}(a)=(u, v)$. The point $b$ in Figure 1 is such that 
$\xi_{S_{k}}(b)=c=1 / 2$. Also, $S_{k}$ is a cutting time for all $a^{\prime} \in(b, v), D_{S_{k}}\left(a^{\prime}\right)=$ $\left[T_{a^{\prime}}^{S_{k}}(c), T_{a^{\prime}}^{S_{Q(k)}}(c)\right]$ for all $a^{\prime} \in(u, v) \backslash\{b\}$, and $S_{k}$ is not a cutting time for all $a^{\prime} \in(u, b)$. Again, for a discussion of these and related details see [14, Chapter 3].

Lemma 5. The set $\mathcal{I}$ is dense in $[\sqrt{2}, 2]$ and is uncountable.

Proof. Let $U \subset[\sqrt{2}, 2]$ be an open interval. Choose $a_{1} \in U \backslash \mathcal{P}$ and a cutting time $n_{1}=S_{k_{1}}\left(a_{1}\right)$ such that $\omega_{n_{1}}\left(a_{1}\right) \subset U$. We can make such a choice due to $\mathcal{P}$ being countable and Lemma 3 . Let $\varepsilon_{1}>0$ and set $J_{1}=\{a \in$ $\omega_{n_{1}}\left(a_{1}\right) \mid n_{1}=S_{k_{1}}(a)$ and $\left.\left|c-c_{n_{1}}(a)\right|<\varepsilon_{1}\right\}$. Then for each $a \in J_{1}$ we see that $n_{1}$ is a cutting time for $T_{a}$ and $\left|c-c_{n_{1}}(a)\right|<\varepsilon_{1}$. Notice that $J_{1}$ is an open subinterval of $U$ (recall Lemma 4 ).

Fix $a_{2} \in J_{1} \backslash \mathcal{P}$. Then $n_{1}=S_{k_{1}}\left(a_{2}\right)$. Set $n_{2}=S_{k_{1}+1}\left(a_{2}\right)$. Choose $0<\varepsilon_{2}<\varepsilon_{1} / 2$ such that $J_{2} \equiv\left\{a \in \omega_{n_{2}}\left(a_{2}\right) \mid n_{2}=S_{k_{1}+1}(a)\right.$ and $\left|c-c_{n_{2}}(a)\right|<$ $\left.\varepsilon_{2}\right\} \subset J_{1}$ and such that the sets $J_{1}$ and $J_{2}$ share no boundary points. Then (again use Lemma 4) for each $a \in J_{2}$ we have $n_{1}=S_{k_{1}}(a), n_{2}=S_{k_{1}+1}(a)$, $\left|c-c_{n_{1}}(a)\right|<\varepsilon_{1}$, and $\left|c-c_{n_{2}}(a)\right|<\varepsilon_{2}$. Also, $\bar{J}_{2}$ is a proper closed subinterval of $\bar{J}_{1}$.

Fix $a_{3} \in J_{2} \backslash \mathcal{P}$. Then $n_{1}=S_{k_{1}}\left(a_{3}\right)$ and $n_{2}=S_{k_{1}+1}\left(a_{3}\right)$. Set $n_{3}=$ $S_{k_{1}+2}\left(a_{3}\right)$. Choose $0<\varepsilon_{3}<\varepsilon_{2} / 2$ such that $J_{3} \equiv\left\{a \in \omega_{n_{3}}\left(a_{3}\right) \mid n_{3}=\right.$ $S_{k_{1}+2}(a)$ and $\left.\left|c-c_{n_{3}}(a)\right|<\varepsilon_{3}\right\} \subset J_{2}$ and the sets $J_{2}$ and $J_{3}$ share no boundary points. Then for each $a \in J_{3}$ we have $n_{1}=S_{k_{1}}(a), n_{2}=S_{k_{1}+1}(a), n_{3}=$ $S_{k_{1}+2}(a),\left|c-c_{n_{1}}(a)\right|<\varepsilon_{1},\left|c-c_{n_{2}}(a)\right|<\varepsilon_{2}$, and $\left|c-c_{n_{3}}(a)\right|<\varepsilon_{3}$. Also, $\bar{J}_{3}$ is a proper closed subinterval of $\bar{J}_{2}$.

Continue this process and set $a_{*}=\bigcap_{n \geq 1} J_{n}$. Remember that if $\liminf _{k \rightarrow \infty} Q_{a_{*}}(k)<\infty$, then (by (3)) there exists some $\delta>0$ such that for infinitely many $k$ we have $\left|c-c_{S_{k-1}}\right|>\delta$. Hence, $\lim _{k \rightarrow \infty} Q_{a_{*}}(k)=\infty$ with $a_{*} \in U$. By varying the choices of $\left\{a_{i}\right\}$ and hence of the sequences of cutting times $\left\{n_{i}\right\}$, one can easily show that $\mathcal{I}$ is uncountable.

Lemma 6. Let $a, a^{\prime} \in[\sqrt{2}, 2]$ and $L>0$. Then for all $x \in[0,1]$,

$$
\left|T_{a}^{L}(x)-T_{a^{\prime}}^{L}(x)\right| \leq\left|a-a^{\prime}\right| \frac{a^{L}-1}{a-1} .
$$

Proof. Clearly, $\left|T_{a}(x)-T_{a^{\prime}}(x)\right| \leq\left|a-a^{\prime}\right|$. Thus,

$$
\begin{aligned}
\left|T_{a}^{L}(x)-T_{a^{\prime}}^{L}(x)\right| \leq & \left|T_{a^{\prime}}\left(T_{a^{\prime}}^{L-1}(x)\right)-T_{a}\left(T_{a^{\prime}}^{L-1}(x)\right)\right| \\
& +\left|T_{a}\left(T_{a^{\prime}}^{L-1}(x)\right)-T_{a}\left(T_{a}^{L-1}(x)\right)\right| \\
\leq & \left|a-a^{\prime}\right|+a\left(\left|T_{a^{\prime}}^{L-1}(x)-T_{a}^{L-1}(x)\right|\right) \\
\leq & \left|a-a^{\prime}\right|\left(1+a+a^{2}+\ldots+a^{L-1}\right)+a^{L}\left|T_{a^{\prime}}^{0}(x)-T_{a}^{0}(x)\right| \\
= & \left|a-a^{\prime}\right| \frac{a^{L}-1}{a-1} .
\end{aligned}
$$


Definition 4. For $a \in[\sqrt{2}, 2]$ and each $k \in \mathbb{N}$ denote by $\omega_{S_{k}}^{\prime}(a)$ that portion of $\omega_{S_{k}}(a)$ (the split being at $a$ ) for which $\xi_{S_{k}}\left(\omega_{S_{k}}^{\prime}(a)\right)$ contains interior points of $D_{S_{k}}(a)$. In Figure $1, \omega_{S_{k}}^{\prime}(a)=(u, a)$.

The next lemma is known (see e.g. [14, Proposition 28]).

Lemma 7. Fix $a \in(\sqrt{2}, 2]$ and let $\omega_{S_{k}}$ and $\omega_{S_{k}}^{\prime}$ be as in Definitions 3 and 4. Then

$$
\lim _{k \rightarrow \infty} \frac{\operatorname{HD}\left(D_{S_{k}}(a), \xi_{S_{k}}\left(\omega_{S_{k}}^{\prime}(a)\right)\right)}{\left|\xi_{S_{k}}\left(\omega_{S_{k}}^{\prime}(a)\right)\right|}=0 .
$$

4. Case 1: $\liminf _{k \rightarrow \infty} Q(k)<\infty$

Proposition 1. Set $\mathcal{H}=\left\{a \in[\sqrt{2}, 2] \mid \liminf _{k \rightarrow \infty} Q_{a}(k)<\infty\right.$ and $a \notin \mathcal{D} \cup \mathcal{P}\}$. Then $\mathcal{H}$ is $\sigma$-porous.

Proof. For each $l \in \mathbb{N}$ set $\mathcal{H}_{l}=\left\{a \in[\sqrt{2}, 2] \backslash \mathcal{D} \mid \liminf _{k \rightarrow \infty} Q_{a}(k)=l\right\}$. Next, we define for each $l \in \mathbb{N}$ and $a \in \mathcal{H}_{l}$ a set $I_{a, l}$ as follows. Fix $l$ and $a \in \mathcal{H}_{l}$. Then for infinitely many $k$ we have $Q_{a}(k)=l$. For each such $k$ we deduce, by (3), that $c_{S_{k-1}} \in\left(z_{l-1}, z_{l}\right] \cup\left[\widehat{z}_{l}, \widehat{z}_{l-1}\right)$. If for infinitely many $k$ we have $c_{S_{k-1}} \in\left(z_{l-1}, z_{l}\right]$, then choose a closed interval with rational endpoints, denoted by $I_{a, l}$, such that $I_{a, l} \subset\left(z_{l}, c\right)$ and $T_{a}^{n}(c) \notin I_{a, l}$ for all $n \in \mathbb{N}$. If for infinitely many $k$ we have $c_{S_{k-1}} \in\left[\widehat{z}_{l}, \widehat{z}_{l-1}\right)$, then choose a closed interval $I_{a, l}$ with rational endpoints such that $I_{a, l} \subset\left(c, \widehat{z}_{l}\right)$ and $T_{a}^{n}(c) \notin I_{a, l}$ for all $n \in \mathbb{N}$. (Since $a \notin \mathcal{D}$ and $T_{a}$ is leo, we can choose such $I_{a, l}$.)

For each $l \in \mathbb{N}$ set $\mathcal{W}_{l}=\left\{I_{a, l} \mid a \in \mathcal{H}_{l}\right\}$. Since the endpoints of all $I_{a, l}$ are rational, for each $l$ the set $\mathcal{W}_{l}$ is countable. For $l \in \mathbb{N}$ and $W \in \mathcal{W}_{l}$ set $\mathcal{H}_{l, W}=\left\{a \in \mathcal{H}_{l} \mid I_{a, l}=W\right\}$.

Claim 1. Each $\mathcal{H}_{l, W}$ is $|W| / 2$-porous.

Proof. Fix $l, W \in \mathcal{W}_{l}$ and $a \in \mathcal{H}_{l, W}$. Thus, $I_{a, l}=W$. Without loss of generality, assume that for infinitely many $k$ we have $c_{S_{k-1}} \in\left[\widehat{z}_{l}, \widehat{z}_{l-1}\right)$ and hence $I_{a, l}=W \subset\left(c, \widehat{z}_{l}\right)$. Say, $W=\left[w_{1}, w_{2}\right]$. Set $W^{\prime}=\xi_{S_{k-1}}^{-1}(W) \cap \omega_{S_{k-1}}(a)$. Say, $W^{\prime}=\left[w_{1}^{\prime}, w_{2}^{\prime}\right]$. Set $\Delta=\left|c_{S_{k-1}}-w_{1}\right|$ and $\Delta^{\prime}=\left|w_{1}^{\prime}-a\right|$. See Figure 2 . From Lemma 2 we find that for large $k, \xi_{S_{k-1}} \mid \omega_{S_{k-1}}(a)$ is almost linear. Hence, $\Delta / \Delta^{\prime} \approx|W| /\left|W^{\prime}\right|$ and therefore $\left|W^{\prime}\right| / \Delta^{\prime} \approx|W| / \Delta \geq|W|$. Thus,

$$
\frac{\left|W^{\prime}\right|}{\Delta^{\prime}}>\frac{|W|}{2} \text {. }
$$

It follows from the definitions of $W=I_{a, l}$ and $\mathcal{H}_{l, W}$ that $W^{\prime} \cap \mathcal{H}_{l, W}=\emptyset$, since for each $a_{0} \in W^{\prime}$ there exists $n=S_{k-1}$ such that $T_{a_{0}}^{n}(c) \in W$. Hence, Claim 1 follows from (6). 


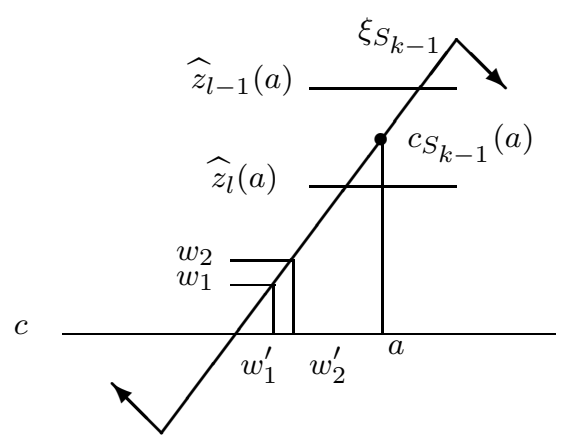

Fig. 2. Construction of $W=I_{a, l}$ and $W^{\prime}$

Lastly, as $\mathcal{H}=\bigcup_{l \in \mathbb{N}} \bigcup_{W \in \mathcal{W}_{l}} \mathcal{H}_{l, W}$, we see that $\mathcal{H}$ is $\sigma$-porous.

5. Substantial and co-substantial cuts. In this section we first give the definition of the key concepts of the next section, the substantial and co-substantial cuts.

We also discuss two algorithms: the greedy and the substantial algorithm. They both can help one to find long stretches and they are behind the dynamics of our proof; in fact, the arguments of the next section can be used to verify that the substantial cut algorithm indeed works. However, we give this idea to help the reader understand the technical details of the next section and the dynamics behind those technicalities.

In this section we assume that $a \in \mathcal{I}$ is fixed, that is, $a \in[\sqrt{2}, 2]$ and $\lim _{k \rightarrow \infty} Q_{a}(k)=\infty$. We will also assume that $\varepsilon$ is a small positive constant.

First we give the definitions of substantial and co-substantial cuts.

Definition 5. We call a cutting time $S_{k}$ a substantial cutting time provided that $\left|c-c_{S_{k}}\right| \leq 3\left|c-c_{S_{k-1}}\right|$.

REMark. As $\lim _{k \rightarrow \infty} Q(k)=\infty$, it follows from (3) and (5) that there are infinitely many substantial cuts. If, additionally, $Q(k)$ is eventually nondecreasing, then it follows from [7, Lemma 2.4] that there exists $K \in \mathbb{N}$ such that for all $k \geq K$, the cut $S_{k}$ is a substantial cut.

Definition 6. We call a cutting time $S_{k}$ a co-substantial cutting time provided that $\left|c-c_{S_{Q(k)}}\right| \leq 3\left|c-c_{S_{k-1}}\right|$.

Next we give an informal discussion of a greedy algorithm. We make the notion precise in Definition 7. Proposition 2 uses the greedy algorithm to produce long stretches. However, long stretches alone are not enough for our porosity result, Theorem 1, and hence we modify the greedy algorithm to obtain the substantial cut algorithm. 
Assume $S_{k_{0}}$ is a cutting time and $J_{0}=\left[z_{k_{0}-1}, c\right]$ and $l_{0}=S_{k_{0}}$. Then $T_{a}^{l_{0}}\left(J_{0}\right)=D_{S_{k_{0}}}=\left[c_{S_{k_{0}}}, c_{S_{Q\left(k_{0}\right)}}\right]$. Our target is to find an $m>l_{0}$ and an interval $I \subset J_{0}$ such that $T_{a}^{m} \mid I$ is monotone and $\left|T_{a}^{m}(I)\right|>\varepsilon$. Since $T_{a}$ is leo it is clear that such an interval exists; the question is how to find it. This is where we can use the greedy algorithm. The interval $J_{0}$ consists of two pieces $J_{0}^{1}=\left[z_{k_{0}}, c\right]$ and $J_{0}^{2}=\left[z_{k_{0}-1}, z_{k_{0}}\right]$ such that $T_{a}^{l_{0}}\left(J_{0}^{1}\right)=\left[c_{S_{k_{0}}}, c\right]$ and $T_{a}^{l_{0}}\left(J_{0}^{2}\right)=\left[c, c_{S_{Q\left(k_{0}\right)}}\right]$. Since $T_{a}^{l_{0}+1}$ is not monotone on $J_{0}$ we need to choose one of these pieces and we make a "greedy choice", that is, we take the bigger piece (i.e., the piece for which $T_{a}^{l_{0}}\left(J_{0}^{i}\right)$ is bigger), and call this piece $J_{1}$. Thus we set $J_{1}=\left[z_{k_{0}}, c\right]$ and $t=k_{0}$ if $\left|c-c_{S_{k_{0}}}\right| \geq\left|c-c_{s_{Q\left(k_{0}\right)}}\right|$ and $J_{1}=\left[z_{k_{0}-1}, z_{k_{0}}\right]$ and $t=Q\left(k_{0}\right)$ otherwise. Clearly, if we set $l_{1}=l_{0}+S_{Q(t+1)}$, then $T_{a}^{l_{1}} \mid J_{1}$ is monotone and $T_{a}^{l_{1}}\left(J_{1}\right)=\left[c_{S_{t+1}}, c_{S_{Q(t+1)}}\right]$. Then $J_{1}$ can be split into two pieces $J_{1}^{1}$ and $J_{1}^{2}$ such that $T_{a}^{l_{1}}\left(J_{1}^{1}\right)=\left[c_{S_{t+1}}, c\right]$ and $T_{a}^{l_{1}}\left(J_{1}^{1}\right)=\left[c, c_{S_{Q(t+1)}}\right]$. Again we are greedy and choose $J_{2}=J_{1}^{1}$ if $\left|c-c_{S_{t+1}}\right| \geq\left|c-c_{S_{Q(t+1)}}\right|$ and $J_{2}=J_{1}^{2}$ otherwise. We keep repeating this procedure to obtain a nested sequence of intervals $J_{0} \supset J_{1} \supset \ldots \supset J_{n} \supset \ldots$ Then for some large $n$ we set $I=J_{n}$ and $m=l_{n}$ to obtain $\left|T_{a}^{m}(I)\right|>\varepsilon$.

Considering $T_{a}^{l_{n}}\left(J_{n}\right)$, there is a corresponding greedy algorithm which describes movements between levels in the Hofbauer tower, corresponding to cutting times. For this algorithm, we are interested in which levels of the tower are visited and hence the algorithm is given as a function from $\mathbb{N}$ into the cutting times $\left\{S_{k}\right\}$. This algorithm is different from the usual action on the tower as described for example in [4].

Definition 7. Fix $m \geq 0$. Define $G_{m}: \mathbb{N} \rightarrow\left\{S_{k}\right\}$ by $G_{m}(1)=S_{m}$ and if $G_{m}(n)=S_{t}$, then

$$
G_{m}(n+1)= \begin{cases}S_{t+1} & \text { if }\left|c-c_{S_{t+1}}\right| \geq\left|c-c_{S_{Q(t+1)}}\right|, \\ S_{Q(t+1)} & \text { else. }\end{cases}
$$

We call this algorithm the greedy algorithm. The name comes from the fact that at each cut we are greedy and take the larger piece of $\left[c_{S_{t+1}}, c_{S_{Q(t+1)}}\right]$.

Lemma 8 is a technical lemma used in Proposition 2 and elsewhere in the paper.

LEMMA 8. Let $S_{k}$ be a substantial cut (resp. a co-substantial cut) with $Q(k)>6$. Then $\left|c-c_{S_{k}}\right|<\left|c-c_{S_{Q(k)}}\right|\left(\right.$ resp. $\left.\left|c-c_{S_{Q(k)}}\right|<\left|c-c_{S_{k}}\right|\right)$.

Proof. We have $\left|c_{S_{k}}-c_{S_{Q(k)}}\right|>8\left|c-c_{S_{k-1}}\right|$, since $Q(k)>6$. The lemma now follows from the definition of a substantial cut (co-substantial cut).

Proposition 2. Let $S_{k}$ be a cutting time and fix $\delta<\min \left\{\left|c-c_{1}\right|\right.$, $\left.\left|c-c_{2}\right|\right\}$. Set $H_{1}=\left[c, c_{S_{Q(k)}}\right]$ and $H_{2}=\left[c, c_{S_{k}}\right]$. Then for $i \in\{1,2\}$, there 
exist a closed interval $I_{i} \subset H_{i}$ and $l_{i} \geq 1$ such that

- $T_{a}^{l_{i}} \mid I_{i}$ is monotone, and

- $\left|T_{a}^{l_{i}}\left(I_{i}\right)\right|>\delta$.

Moreover, when $S_{k}$ is a substantial cut with $Q(k)>6$, then $1 \leq l_{1} \leq S_{k-1}$.

Proof. For $i=1$, set $G=G_{S_{Q(k)}}$ and for $i=2$, set $G=G_{S_{k}}$.

Claim 1. Fix $i \in\{1,2\}$. If $G(n)=S_{m}$ with $Q(m+1)>1$, then $\left|c-c_{G(n)}\right|<\left|c-c_{G(n+1)}\right|$.

Proof. Since $Q(m+1)>1$, we have $S_{Q(m+1)}>S_{1}=2$ and hence $a^{S_{Q(m+1)}}>2$. But $a^{S_{Q(m+1)}}>2$ implies that $\left|c_{S_{m+1}}-c_{S_{Q(m+1)}}\right|>2\left|c-c_{S_{m}}\right|$. It is now easy to check that the claim holds by the definition of $G$.

Assume that $i=1$; the case $i=2$ is similar. If, when applying the greedy algorithm $G$, we arrive at a level of the tower $S_{m}$ with $Q(m+1) \in\{0,1\}$ then we are done since either $\left|c-c_{1}\right|$ or $\left|c-c_{2}\right|$ is contained in $D_{S_{m+1}}$.

Since $\lim _{k \rightarrow \infty} Q(k)=\infty$, there exists $t \geq 1$ such that $\left|c-c_{S_{Q(k)+t}}\right|<$ $\left|c-c_{S_{Q}(k)}\right|$. Hence, if we have the condition " $Q(m+1)>1$ " when applying the greedy algorithm, then (due to Claim 1) we cannot get to a level in the tower above $S_{Q(k)+t}$. Thus, we arrive at any level of the tower at most once until we arrive at $D_{2}$ or $D_{1}$, in which case we are done. If $S_{k}$ is a substantial cut and $Q(k)>6$, then by Claim 1 and Lemma 8 we cannot return to level $S_{k}$ and hence cannot get above this level. Therefore $l_{1} \leq S_{k-1}$.

As previously remarked, for our porosity estimate we need more than just to find a long stretch. Assume that we have a small number $\tilde{\eta}>0$ given in advance and we also have a $k_{0}$ such that $S_{k_{0}}$ is a substantial cut and we want to find an interval $I \subset\left[c, c_{S_{Q\left(k_{0}\right)}}\right]$ and an $l \geq 1$ such that $\left.T_{a}^{l}\right|_{I}$ is monotone, $\left|T_{a}^{l}(I)\right|>\varepsilon$ and $|I|>\widetilde{\eta} \widetilde{E}_{I}$ where $\widetilde{E}_{I}$ is the length of the shortest closed interval containing both $I$ and $c_{S_{k_{0}}}$. For our porosity estimates we need $|I| \geq \widetilde{\eta} \widetilde{E}_{I}$, which we call the metric assumption for our porosity estimates; this assumption roughly means that we not only want an interval on which we have a long stretch, but we want the length of this interval to be sufficiently large, compared to its distance from $c_{S_{k_{0}}}$. Of course, the best situation is when this interval is a central branch (which we can have if $a \notin \mathcal{I}$ ), but for $a \in \mathcal{I}$ finding such intervals is more difficult. In an algorithm which we call the substantial cut algorithm, as in the greedy algorithm, we will define a nested sequence of intervals $J_{0} \supset J_{1} \supset \ldots \supset J_{n} \supset \ldots$ such that for a sequence $l_{0}=S_{k_{0}}<l_{1}<\ldots<l_{n}<\ldots, T_{a}^{l_{n}} \mid J_{n}$ is monotone and $T_{a}^{l_{n}}\left(J_{n}\right)$ corresponds to a cutting time level of the Hofbauer tower, $J_{0}=\left[\alpha_{0}, \beta_{0}\right]$ with $T_{a}^{S_{k_{0}}}\left(\alpha_{0}\right)=c_{S_{k_{0}}}$ and $T_{a}^{S_{k_{0}}}\left(\beta_{0}\right)=c_{S_{Q\left(k_{0}\right)}}$. For a large value of $n$ we will be able to choose $I=T_{a}^{S_{k_{0}}}\left(J_{n}\right)$. 
To understand the dynamics behind the next technical section we need to see how the greedy algorithm is modified, that is, how we define $J_{n+1}$ by selecting a proper piece of $J_{n}$.

Assume $J_{n}=\left[\alpha_{n}, \beta_{n}\right]$; we chose our notation so that $\alpha_{n}$ is closer to $\alpha_{0}$ than $\beta_{n}$. To satisfy our metric assumption we will have to control our greed and sometimes we will have to keep the shorter piece of $J_{n}$ in order to stay sufficiently close to $\alpha_{0}$. To be more precise assume that $T_{a}^{l_{n}}\left(J_{n}\right)=$ $\left[c_{S_{t}}, c_{S_{Q(t)}}\right]$ for an integer $t$. If $T_{a}^{l_{n}}\left(\alpha_{n}\right)=c_{S_{Q(t)}}$ we say that we are in $a$ co-active situation. Otherwise, when $T_{a}^{l_{n}}\left(\alpha_{n}\right)=c_{S_{t}}$, we are not in a co-active situation. Choose $\gamma_{n} \in\left[\alpha_{n}, \beta_{n}\right]$ such that $T_{a}^{l_{n}}\left(\gamma_{n}\right)=c$. In the non-co-active case we check whether the cut at $\left[c_{S_{t}}, c_{S_{Q(t)}}\right]$ is substantial or not; if it is then we set $J_{n+1}=\left[\gamma_{n}, \beta_{n}\right]$ (that is, we are greedy and keep the longer piece; recall Lemma 8); if it is not a substantial cut then we set $J_{n+1}=\left[\alpha_{n}, \gamma_{n}\right]$ (that is, in order to satisfy the metric assumption we choose the piece closer to $\alpha_{0}$ even if it is smaller than the other piece; since we do not have a substantial cut this smaller piece is still relatively long). In the co-active case we check whether the cut at $\left[c_{S_{t}}, c_{S_{Q(t)}}\right]$ is co-substantial or not; if it is then we set $J_{n+1}=\left[\gamma_{n}, \beta_{n}\right]$ (that is, we are greedy and keep the longer piece); if it is not a co-substantial cut then we set $J_{n+1}=\left[\alpha_{n}, \gamma_{n}\right]$ (that is, in order to satisfy the metric assumption we choose the piece closer to $\alpha_{0}$ even if it is smaller than the other piece; since we do not have a co-substantial cut this smaller piece is still relatively long).

Next we give a formal definition of the substantial cut algorithm.

Let $k_{0} \in \mathbb{N}$ be fixed and assume $S_{k_{0}}$ is a substantial cut. Set $\Gamma(0)=0$, $J_{0}=\left[z_{k_{0}-1}, c\right]$ and $l_{0}=S_{k_{0}}$. (The auxiliary function $\Gamma$ tells us whether we are at a co-active $(\Gamma=1)$ or a non-co-active $(\Gamma=0)$ cut.) Then $T_{a}^{l_{0}}\left(J_{0}\right)=$ $D_{S_{k_{0}}}=\left[c_{S_{k_{0}}}, c_{S_{Q\left(k_{0}\right)}}\right]$, and $T_{a}^{l_{0}} \mid J_{0}$ is monotone. Let $J_{1} \subset J_{0}$ be such that $T_{a}^{l_{0}}\left(J_{1}\right)=\left[c, c_{S_{Q\left(k_{0}\right)}}\right]$. Set $k_{1}=Q\left(k_{0}\right)+1$ and $l_{1}=S_{k_{0}}+S_{Q\left(k_{1}\right)}$. Then $T_{a}^{l_{1}}\left(J_{1}\right)=D_{S_{k_{1}}}=\left[c_{S_{k_{1}}}, c_{S_{Q\left(k_{1}\right)}}\right]$, and $T_{a}^{l_{1}} \mid J_{1}$ is monotone. Set $\Gamma(1)=1$.

Assume that $n \geq 1$ and that we have constructed finite sequences $\{\Gamma(i)\}_{i=0}^{n},\left\{k_{i}\right\}_{i=0}^{n},\left\{l_{i}\right\}_{i=0}^{n}$, and closed nested intervals $J_{0} \supset J_{1} \supset \ldots \supset J_{n}$ such that

- $T_{a}^{l_{i}} \mid J_{i}$ is monotone for $0 \leq i \leq n$,

- $T_{a}^{l_{i}}\left(J_{i}\right)=D_{S_{k_{i}}}$ for $0 \leq i \leq n$,

- $l_{i}=l_{i-1}+S_{Q\left(k_{i}\right)}$ for $1 \leq i \leq n$,

- $k_{i} \in\left\{k_{i-1}+1, Q\left(k_{i-1}\right)+1\right\}$ for $1 \leq i \leq n$,

- $k_{i}=k_{i-1}+1 \Rightarrow T_{a}^{l_{i-1}}\left(J_{i}\right)=\left[c, c_{S_{k_{i-1}}}\right]$ for $1 \leq i \leq n$,

- $k_{i}=Q\left(k_{i-1}\right)+1 \Rightarrow T_{a}^{l_{i-1}}\left(J_{i}\right)=\left[c, c_{S_{Q\left(k_{i-1}\right)}}\right]$ for $1 \leq i \leq n$, and

- $\Gamma(i)$ tells us whether the cut at $S_{k_{i}}$ is co-active or not. 
We want to define $k_{n+1}, l_{n+1}$, and $J_{n+1}$. There are two options, which we call Option A and Option B.

Option A. Set $k_{n+1}=k_{n}+1$ and $l_{n+1}=l_{n}+S_{Q\left(k_{n+1}\right)}$. Let $J_{n+1} \subset J_{n}$ be such that $T_{a}^{l_{n+1}} \mid J_{n+1}$ is monotone and $T_{a}^{l_{n+1}}\left(J_{n+1}\right)=D_{S_{k_{n+1}}}$.

Option B. Set $k_{n+1}=Q\left(k_{n}\right)+1$ and $l_{n+1}=l_{n}+S_{Q\left(k_{n+1}\right)}$. Let $J_{n+1} \subset J_{n}$ be such that $T_{a}^{l_{n+1}} \mid J_{n+1}$ is monotone and $T_{a}^{l_{n+1}}\left(J_{n+1}\right)=D_{S_{k_{n+1}}}$.

If $\Gamma(n)=0$ and $S_{k_{n}}$ is substantial (resp. not substantial) then set $\Gamma(n+1)$ $=1$ and use Option B (resp. set $\Gamma(n+1)=0$ and use Option A) to define $k_{n+1}, l_{n+1}$, and $J_{n+1}$.

If $\Gamma(n)=1$ and $S_{k_{n}}$ is co-substantial (resp. not co-substantial) then set $\Gamma(n+1)=1$ and use Option A (resp. set $\Gamma(n+1)=0$ and use Option B) to define $k_{n+1}, l_{n+1}$, and $J_{n+1}$.

The above definition of $\Gamma(n+1)$ explains our name for the function $\Gamma$. If $\Gamma(n)=1$, then we need to check whether the cut at $S_{k_{n}}$ is co-substantial or not (co-active case). If $\Gamma(n)=0$, then we need to check whether the cut at $S_{k_{n}}$ is substantial or not (non-co-active case).

If for some $j$ we have $\left|D_{S_{k_{j}}}\right|>\varepsilon$, then the algorithm terminates at this step and $J_{j}$ can be chosen as $I^{\prime}$, and $l_{j}$ as $m$.

It is worthwhile to compare the substantial cut algorithm and the greedy algorithm after this formal definition.

Assume $k_{n}, l_{n}$, and $J_{n}$ are defined. Recall that $D_{S_{k_{n}}}=\left[c_{S_{k_{n}}}, c_{S_{Q\left(k_{n}\right)}}\right]$. During the greedy algorithm we use Option A if $\left|c-c_{S_{k_{n}}}\right| \geq\left|c-c_{S_{Q\left(k_{n}\right)}}\right|$ and Option B otherwise. This means that we are greedy, we always want to follow the "larger piece" at each cut.

In the substantial cut algorithm, to satisfy the metric assumption (that is, we need the long stretch relatively close to $c$ ) we allow the use of Option A for nonsubstantial cuts. At nonsubstantial cuts it may still happen that $\left|c-c_{S_{k_{n}}}\right|<\left|c-c_{S_{Q\left(k_{n}\right)}}\right|$, but at these steps, to obtain the metric estimate, we limit our "greed" and choose the piece which stays close to $c$. Studying the proof of Lemma 12 of Section 6 one can verify that being nongreedy at these steps yields the desired estimate for the metric assumption.

Finally, we show that substantial cuts are interesting for other reasons as well. It is obvious that at a cutting time $\left|D_{S_{k}}\right|$ can be arbitrarily small. On the other hand, for substantial cuts we have:

Lemma 9. Let $S_{k}$ be a substantial cut and set $\delta=\min \left\{\left|c-c_{j}\right| \mid 1 \leq j\right.$ $\leq 6\}$. Then

$$
\left|D_{S_{k}}\right| \geq a^{-S_{k-1}} \delta
$$

Proof. If $Q(k) \leq 6$, then (7) follows from (5), the definition of $\delta$, and $a^{-S_{k-1}}<1$. Assume that $Q(k)>6$. Then, from Proposition 2, there exist 
$L \subset D_{S_{k}}$ and $l \leq S_{k-1}$ such that $T_{a}^{l} \mid L$ is monotone and $\left|T_{a}^{l}(L)\right|>\delta$. Now, $L \subset D_{S_{k}}, a^{l}|L| \geq \delta$, and $l \leq S_{k-1}$ imply the result.

6. Tools for the case $\lim _{k \rightarrow \infty} Q(k)=\infty$. Throughout this section $a \in \mathcal{I}$ is fixed; recall $a \in(\sqrt{2}, 2)$. In this section we give the technical details of the estimates corresponding to this case. Behind the "dynamics" of our argument there is the substantial cut algorithm. Our argument is based on induction; the key result of this section is Proposition 3 which roughly states that if we can find a sufficiently long stretch with good metric estimates for all suitable "lower level" substantial and co-substantial cuts in the Hofbauer tower, then we can find the required long stretch at the "next level" as well.

Choose $K_{1} \in \mathbb{N}$ such that

- $k>K_{1}$ and $l^{\prime} \geq \min \{Q(k+1), Q(Q(k)+1)\}$ imply that $S_{Q\left(l^{\prime}\right)} \geq 20$,

- $k>K_{1}$ implies that $Q(k)>100$.

As $a>\sqrt{2}$, we have $a^{S_{Q\left(l^{\prime}\right)}}>2^{10}>1000$ for $l^{\prime}$ satisfying the above inequality. Since $a \notin \mathcal{P}$, there exists $\varepsilon_{1}>0$ such that $\left|c_{S_{k}}-c_{S_{Q(k)}}\right|>\varepsilon_{1}$ for $k \leq K_{1}$. Also, throughout this section we assume that $0<\varepsilon<\min \left\{\left|c-c_{1}\right|,\left|c-c_{2}\right|\right.$, $\left.\varepsilon_{1} / 2\right\}$ is fixed and $\eta \in(0,1 / 2)$.

In the next definition we introduce auxiliary points $y_{k}$ and $\bar{y}_{k}$ for substantial cuts. These points will help us in our induction for the estimates needed for the metric assumption; we will use them to show that if we have good metric properties at "lower levels" of the tower then we have good properties at the "next level" as well.

DEFinition 8. If $S_{k}$ is a substantial cut for $T_{a}$, define $y_{k}$ to be the unique point on the same side of $c$ as $c_{S_{k}}$ such that $\left|y_{k}-c\right|=4 a^{-S_{Q(k)}}\left|c-c_{S_{Q(k)}}\right|$. The point $\bar{y}_{k}$ is defined similarly to $y_{k}$ but with $\left|\bar{y}_{k}-c\right|=2\left|y_{k}-c\right|=$

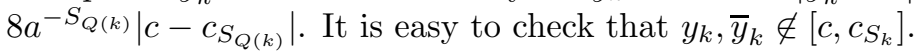

The next definition will give our metric assumption (based on the auxiliary point $y_{k}$ ) which we can use in our induction. The $\varepsilon$ - $\eta$-good substantial cuts will provide long stretches with good "metric properties".

Definition 9. A substantial cut $S_{k}$ is said to be $\varepsilon$ - $\eta$-good provided that there exist $I \subset\left[c, c_{S_{Q(k)}}\right]$ and $l \geq 1$ such that $T_{a}^{l} \mid I$ is monotone, $\left|T_{a}^{l}(I)\right|>\varepsilon$, and $|I| \geq \eta E_{I}$, where $E_{I}$ is the length of the shortest closed interval containing both $y_{k}$ and $I$. (See Figure 3.)

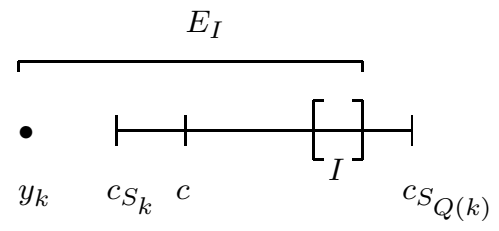

Fig. 3. Construction of $E_{I}$ 
Definition 10. A substantial cut $S_{k}$ is strongly $\varepsilon-\eta$-good provided there exist $I \subset\left[c, c_{S_{Q(k)}}\right]$ and $l \geq 1$ such that $T_{a}^{l} \mid I$ is monotone, $\left|T_{a}^{l}(I)\right|>\varepsilon$, and $|I| \geq \eta \bar{E}_{I}$, where $\bar{E}_{I}$ is the length of the shortest closed interval containing both $\bar{y}_{k}$ and $I$.

The next three definitions are the co-substantial versions of the previous two.

Definition 11. If $S_{k}$ is a co-substantial cut for $T_{a}$, define $y_{k}^{\prime}$ to be the unique point on the same side of $c$ as $c_{S_{Q(k)}}$ with $\left|y_{k}^{\prime}-c\right|=4 a^{-S_{Q(k)}}\left|c-c_{S_{k}}\right|$. The point $\bar{y}_{k}^{\prime}$ is defined similarly to $y_{k}^{\prime}$ but satisfies $\left|\bar{y}_{k}^{\prime}-c\right|=2\left|y_{k}^{\prime}-c\right|=$ $8 a^{-S_{Q(k)}}\left|c-c_{S_{k}}\right|$. Again, it is easy to check that $y_{k}^{\prime}, \bar{y}_{k}^{\prime} \notin\left[c, c_{S_{Q(k)}}\right]$.

Definition 12. A co-substantial cut $S_{k}$ is said to be $\varepsilon$ - $\eta$-good provided there exist $I \subset\left[c, c_{S_{k}}\right]$ and $l \geq 1$ such that $T_{a}^{l} \mid I$ is monotone, $\left|T_{a}^{l}(I)\right|>\varepsilon$, and $|I| \geq \eta E_{I}$, where $E_{I}$ is the length of the shortest closed interval containing both $y_{k}^{\prime}$ and $I$.

Definition 13. A co-substantial cut $S_{k}$ is strongly $\varepsilon-\eta$-good provided there exist $I \subset\left[c, c_{S_{k}}\right]$ and $l \geq 1$ such that $T_{a}^{l} \mid I$ is monotone, $\left|T_{a}^{l}(I)\right|>\varepsilon$, and $|I| \geq \eta \bar{E}_{I}$, where $\bar{E}_{I}$ is the length of the shortest closed interval containing both $\bar{y}_{k}^{\prime}$ and $I$.

Lemma 10. Let $S_{k}$ be a substantial cut. Set $p_{0}=Q(k)+1$ and $q_{0}=$ $Q\left(p_{0}\right)$. If $S_{p_{0}}$ is not a co-substantial cut, then there exists $l \geq 1$ such that $S_{q_{0}+l}$ is a substantial cut, $L \equiv S_{q_{0}+l}=S_{q_{0}}+S_{Q\left(q_{0}+1\right)}+\ldots+S_{Q\left(q_{0}+l\right)} \leq S_{Q(k)}$, and $S_{q_{0}+l^{\prime}}$ is not a substantial cut for $1 \leq l^{\prime}<l$.

What is the dynamics behind this lemma? In the next figure we show some levels of the Hofbauer tower. The top level corresponds to the cut at $S_{k}$. To satisfy our metric assumptions in the substantial cut algorithm we want to stay "close to" $c_{S_{k}}$, marked by an arrow. Of course, due to the substantial cut, we need to throw away the small piece containing $c_{S_{k}}$, and we follow the iterated $T_{a}$ images of $c$ instead (the other point marked by an arrow on the top level). At level $S_{p_{0}}$ we mark by an arrow the $T_{a}^{S_{q_{0}}}$ image of $c$, which actually equals $c_{S_{Q\left(p_{0}\right)}}$. This is the "co-endpoint" of the Hofbauer tower level at $S_{p_{0}}$. We now drop down to the bottom level in the figure, $S_{Q\left(p_{0}\right)}$, and we also picture the image of the level $S_{p_{0}}$ as a subset of the bottom level. By our assumption we do not have a co-substantial cut at level $S_{p_{0}}$, that is, the "co-piece" of length $\Delta$ is sufficiently long. Now, starting from the bottom level, we move up in the tower until at level $S_{q_{0}+l}$ we have again a substantial cut. At the nonsubstantial cuts we just simply follow the piece which contains the $c_{S_{q_{0}}+j}$ non- "co-endpoint", $j=1, \ldots, l-1$; these endpoints are marked by an arrow again. Finally, we have a substantial cut at level $S_{q_{0}+l}$, and the whole procedure starts again... 


$$
\begin{aligned}
& c_{S_{k} c}^{4} c_{S_{Q(k)}}
\end{aligned}
$$

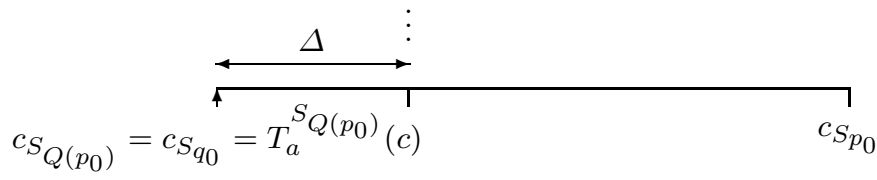

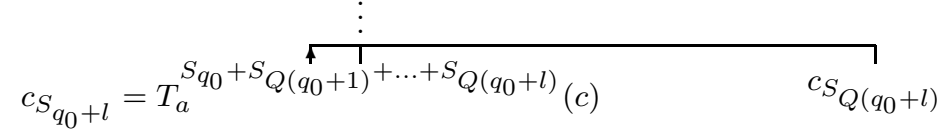

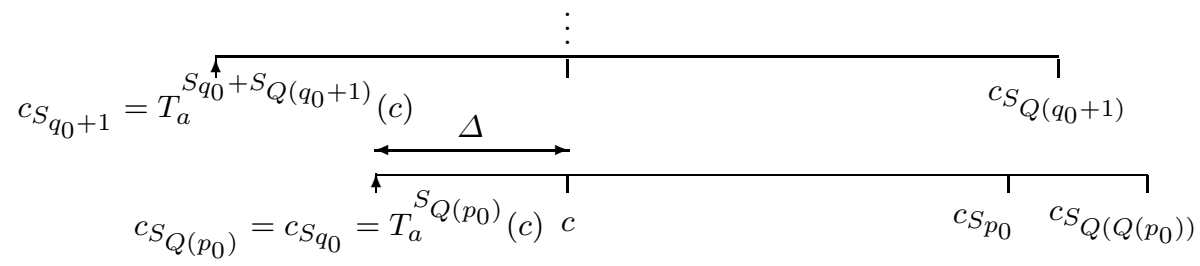

Fig. 4. Some Hofbauer tower levels

Proof of Lemma 10. Set $\Delta=\left|c-c_{S_{Q(Q(k)+1)}}\right|=\left|c-c_{S_{q_{0}}}\right|$. From (4), $q_{0}=Q(Q(k)+1) \leq Q(k)$. Since $S_{p_{0}}$ is not a co-substantial cut, it follows that

$$
\Delta>3\left|c-c_{S_{Q(k)}}\right|
$$

Hence, $q_{0}<Q(k)$, else $q_{0}=Q(k)$ and $\Delta=\left|c-c_{S_{Q(k)}}\right|$, contradicting (8). If there does not exist such an $l$, then $\left|c-c_{S_{q_{0}+1}}\right|>3\left|c-c_{S_{q_{0}}}\right|=3 \Delta$, $\left|c-c_{S_{q_{0}+2}}\right|>3\left|c-c_{S_{q_{0}+1}}\right|>3^{2} \Delta, \ldots,\left|c-c_{S_{Q(k)}}\right|>3^{Q(k)-q_{0}} \Delta$, again contradicting (8).

LemMa 11. Let $S_{k}$ be a co-substantial cut with $Q(k) \geq 6$. Set $p_{0}=k+1$ and $q_{0}=Q\left(p_{0}\right)$. If $S_{p_{0}}$ is not a co-substantial cut, then there exists $l \geq 1$ such that $S_{q_{0}+l}$ is a substantial cut, $L=S_{q_{0}+l}=S_{q_{0}}+S_{Q\left(q_{0}+1\right)}+\ldots+S_{Q\left(q_{0}+l\right)} \leq$ $S_{Q(k)}$, and $S_{q_{0}+l^{\prime}}$ is not a substantial cut for $1 \leq l^{\prime}<l$.

Proof. Set $\Delta=\left|c-c_{S_{k}}\right|$. Since $S_{k}$ is a co-substantial cut and $Q(k) \geq 6$, we have $\Delta \geq\left|c-c_{S_{k-1}}\right|$ and thus, from $(3), q_{0}=Q(k+1) \leq Q(k)$. Next, $S_{p_{0}}$ not a co-substantial cut implies that $\left|c-c_{S_{q_{0}}}\right|>3 \Delta$, and $S_{k}$ a co-substantial cut implies that $\left|c-c_{S_{Q(k)}}\right| \leq \Delta$; thus $q_{0}=Q(k+1)<Q(k)$. Again, if there does not exist such an $l$, then $\Delta \geq\left|c-c_{S_{Q(k)}}\right| \geq 3^{Q(k)-q_{0}}\left|c-c_{S_{q_{0}}}\right|>$ $3^{Q(k)-q_{0}} 3 \Delta$, a contradiction.

The next lemma shows that if in our substantial cut algorithm a substantial cut is not followed by a co-substantial cut and the later substantial cut (which exists by Lemmas 10 and 11 ) is $\varepsilon$ - $\eta$-good then the original substantial cut is strongly $\varepsilon$ - $\eta$-good. 
Lemma 12. Assume that $k>K_{1}$. If $S_{k}$ is a substantial cut, $S_{p_{0}}$ is not a co-substantial cut, where $p_{0}=Q(k)+1$, and $S_{q_{0}+l}$ is $\varepsilon-\eta$-good, where $q_{0}=Q\left(p_{0}\right)$ and $l$ is as in Lemma 10, then the cut at $S_{k}$ is strongly $\varepsilon-\eta$-good.

Of course, the above lemma has a "co-substantial" version as well:

Lemma 13. Assume that $k>K_{1}$. If $S_{k}$ is a co-substantial cut, $S_{p_{0}}$ is not a co-substantial cut, where $p_{0}=k+1$, and $S_{q_{0}+l}$ is $\varepsilon$ - $\eta$-good, where $q_{0}=Q\left(p_{0}\right)$ and $l$ is as in Lemma 11 , then the cut at $S_{k}$ is strongly $\varepsilon-\eta$-good.

Next we give the proof of Lemma 12 and in parenthetical remarks we show what should be modified for the proof of Lemma 13.

P r o o f. We use the notation of Lemma 10 (Lemma 11 for proving Lemma 13). Thus, $S_{q_{0}}<S_{Q(k)}$ and $l \geq 1$ is minimal such that $S_{q_{0}+l}$ is a substantial cut.

Set $C=c_{S_{Q(k)}}$. (To prove Lemma 13 set $C=c_{S_{k}}$.) Let $h=|c-C|$ and for $0 \leq i \leq l$, set $h_{i}=\left|c-c_{S_{q_{0}+i}}\right|$. Then

$$
3 h<h_{0} \text { and } 3 h_{l^{\prime}-1}<h_{l^{\prime}}
$$

for $1 \leq l^{\prime} \leq l-1$. From the definition of the $z_{k}$ 's (see (1)) and the definition of $q_{0}$ we deduce that either $[c, C] \ni z_{q_{0}}$ or $[c, C] \ni \widehat{z}_{q_{0}}$. Recall that $L=$ $S_{q_{0}+l}$. Without loss of generality, assume that $[c, C] \ni z_{q_{0}}$ and therefore that $[c, C] \ni z_{q_{0}+l-1}$. Recall that $T_{a}^{L}$ maps $\left[c, z_{q_{0}+l-1}\right]$ onto $\left[c_{S_{q_{0}+l}}, c_{S_{Q\left(q_{0}+l\right)}}\right]$ in a one-to-one manner with $T_{a}^{L}\left(z_{q_{0}+l-1}\right)=c_{S_{Q\left(q_{0}+l\right)}}$. Denote by $\phi$ the linear mapping that is the extension of $T_{a}^{L} \mid\left[c, z_{q_{0}+l-1}\right]$ onto $\mathbb{R}$. It is clear that the absolute value of the slope of $\phi$ is $a^{L}$. Clearly, $z_{q_{0}+l}=\phi^{-1}(c)$ and $\left|c-z_{q_{0}+l}\right|=a^{-L}\left|c-c_{S_{q_{0}+l}}\right|$. Set $w=\phi^{-1}\left(y_{q_{0}+l}\right)$; recall Definition 8 for $y_{q_{0}+l}$. Then $w$ is on the same side of $c$ as $\bar{y}_{k}$. (In the proof of Lemma 13, $w$ is on the same side of $c$ as $\bar{y}_{k}^{\prime}$.)

Using $\left|c-c_{S_{q_{0}+l}}\right|+\left|c-c_{S_{Q\left(q_{0}+l\right)}}\right|=a^{S_{Q\left(q_{0}+l\right)}}\left|c-c_{S_{q_{0}+l-1}}\right|$ and $\left|c-c_{S_{q_{0}+l}}\right| \leq$ $3\left|c-c_{S_{q_{0}+l-1}}\right|$, we have

$$
\begin{aligned}
h_{l-1} a^{S_{Q\left(q_{0}+l\right)}} & =\left|c-c_{S_{Q\left(q_{0}+l\right)}}\right|+\left|c-c_{S_{q_{0}+l}}\right| \\
& \leq\left|c-c_{S_{Q\left(q_{0}+l\right)}}\right|\left(1+\frac{3 a^{-S_{Q\left(q_{0}+l\right)}}}{1-3 a^{-S_{Q\left(q_{0}+l\right)}}}\right) \\
& =\left|c-c_{S_{Q\left(q_{0}+l\right)}}\right| \frac{1}{1-3 a^{-S_{Q\left(q_{0}+l\right)}}} .
\end{aligned}
$$

Hence,

$$
h_{l-1} a^{S_{Q\left(q_{0}+l\right)}}\left(1-3 a^{-S_{Q\left(q_{0}+l\right)}}\right) \leq\left|c-c_{S_{Q\left(q_{0}+l\right)}}\right| \leq h_{l-1} a^{S_{Q\left(q_{0}+l\right)}} .
$$

From the definition of $y_{q_{0}+l}$ and (10) we get

$$
\left|c-y_{q_{0}+l}\right|=4 a^{-S_{Q\left(q_{0}+l\right)}}\left|c-c_{S_{Q\left(q_{0}+l\right)}}\right| \geq 4 h_{l-1}\left(1-3 a^{-S_{Q\left(q_{0}+l\right)}}\right) .
$$


Thus (use $\left|c-y_{q_{0}+l}\right|=a^{S_{q_{0}+l}}\left|w-z_{q_{0}+l}\right|$ and $L=S_{q_{0}+l}$ )

$$
\left|w-z_{q_{0}+l}\right| \geq 4 a^{-L} h_{l-1}\left(1-3 a^{\left.-S_{Q\left(q_{0}+l\right)}\right)} .\right.
$$

On the other hand,

$$
\begin{aligned}
\left|c-z_{q_{0}+l}\right| & =a^{-L}\left|c-c_{S_{q_{0}+l}}\right| \leq a^{-L} \frac{3 a^{-S_{Q\left(q_{0}+l\right)}}}{1-3 a^{-S_{Q\left(q_{0}+l\right)}}}\left|c-c_{S_{Q\left(q_{0}+l\right)}}\right| \\
& \leq a^{-L} \frac{3 a^{-S_{Q\left(q_{0}+l\right)}}}{1-3 a^{-S_{Q\left(q_{0}+l\right)}}} a^{S_{Q\left(q_{0}+l\right)}} h_{l-1} \\
& =a^{-L} \frac{3}{1-3 a^{-S_{Q\left(q_{0}+l\right)}}} h_{l-1} .
\end{aligned}
$$

Thus, using (9), (11) and (12), we have

$$
\begin{aligned}
|c-w| & \geq a^{-L} h_{l-1}\left(4\left(1-3 a^{-S_{Q\left(q_{0}+l\right)}}\right)-\frac{3}{1-3 a^{-S_{Q\left(q_{0}+l\right)}}}\right) \\
& \geq a^{-L} h_{l-1}\left(4 \cdot 0.99-\frac{3}{0.99}\right)>0.9 a^{-L} h_{l-1} \\
& \geq 0.9 \cdot 3^{l} a^{S_{Q(k)}-L} a^{-S_{Q(k)}} h \equiv A .
\end{aligned}
$$

Here we have used the fact that $q_{0}+l \geq \min \{Q(k), Q(k+1), Q(Q(k)+1)\}$ and hence that $S_{Q\left(q_{0}+l\right)} \geq 20$, i.e., $a^{-S_{Q\left(q_{0}+l\right)}}<0.001$. More succinctly,

$$
|c-w| \geq 0.9 \cdot 3^{l} a^{S_{Q(k)}-L} a^{-S_{Q(k)}} h \equiv A .
$$

Since the assumptions of Lemma 10 hold (in the proof of Lemma 13 we use Lemma 11), we have $L=S_{q_{0}+l} \leq S_{Q(k)}$. If $L<S_{Q(k)}$, then $S_{Q(k)}-L \geq$ $S_{Q\left(q_{0}+l+1\right)}>20$, since $q_{0}+l+1 \geq \min \{Q(k+1), Q(Q(k)+1)\}$ and $k>K_{1}$. Hence if $S_{Q(k)} \neq L$, then $S_{Q(k)}-L \geq 20$. We use this fact in the next claim.

Claim 1. We have $\bar{y}_{k} \in[w, c]$.

Proof. First assume that either $l \geq 2$, or that $S_{Q(k)} \neq L$. Then, by (13), $|c-w| \geq A>8 a^{-S_{Q(k)} h}=\left|\bar{y}_{k}-c\right|$ and therefore $\bar{y}_{k} \in[w, c]$.

Next assume $l=1$ and $S_{Q(k)}=L$. Then $c_{S_{q_{0}+l}}=c_{S_{Q(k)}}$ and $\left|c-c_{S_{q_{0}+l}}\right|$ $\leq h$ (here we have equality when $S_{k}$ is a substantial cut and strict inequality when $S_{k}$ is a co-substantial cut). Hence, $\left|c-z_{q_{0}+l}\right| \leq a^{-L} h$. From (11) we have $\left|w-z_{q_{0}+l}\right| \geq 4 a^{-L} \cdot 0.99 h_{0} \geq 12 a^{-L} \cdot 0.99 h>11 a^{-L} h$. Lastly, $\left|c-z_{q_{0}+l}\right| \leq a^{-L} h$ and $\left|w-z_{q_{0}+l}\right|>11 a^{-L} h$ imply that $|c-w| \geq 10 a^{-L} h=$ $10 a^{-S_{Q(k)}} h$. Thus, $\left|c-\bar{y}_{k}\right|=8 a^{-S_{Q(k)}} h<|c-w|$.

(The proof of Claim 1 with $\bar{y}_{k}$ replaced by $\bar{y}_{k}^{\prime}$ gives the next claim, which is used in the proof of Lemma 13.

Claim 2. We have $\bar{y}_{k}^{\prime} \in[w, c]$.)

By assumption $S_{q_{0}+l}$ is $\varepsilon$ - $\eta$-good. Hence, choose $I \subset\left[c, c_{S_{Q\left(q_{0}+l\right)}}\right]$ and $l^{\prime} \geq 1$ with $T_{a}^{l^{\prime}} \mid I$ monotone, $\left|T_{a}^{l^{\prime}}\right|>\varepsilon$, and $|I| \geq \eta E_{I}$, where $E_{I}$ is the 
length of the shortest closed interval containing both $y_{q_{0}+l}$ and $I$. Set $J=$ $\phi^{-1}(I)$.

Then $J \subset\left[z_{q_{0}+l}, c_{S_{Q(k)}}\right] \subset\left[c, c_{S_{Q(k)}}\right]$. From linearity of $\phi,|I| \geq \eta E_{I}$, and the definition of $w$ we obtain $|J| \geq \eta \bar{E}_{J}$, where $\bar{E}_{J}$ is the length of the shortest closed interval containing both $w$ and $J$. Let $E_{J}$ be the length of the shortest closed interval containing both $\bar{y}_{k}$ and $J$. From $E_{J}<\bar{E}_{J}$ and $|J| \geq \eta \bar{E}_{J}$, we have $|J| \geq \eta E_{J}$. Lastly, since $\left|T_{a}^{L+l^{\prime}}(J)\right|=\left|T_{a}^{l^{\prime}}(I)\right|>\varepsilon$ and $T_{a}^{L+l^{\prime}} \mid J$ is monotone, it follows that $S_{k}$ is strongly $\varepsilon-\eta$-good.

(In the proof of Lemma 13 an argument similar to the above paragraph is used with $J \subset\left[c, c_{S_{k}}\right]$.)

The next two lemmas are the versions of Lemmas 12 and 13 which work in the case when a substantial or a co-substantial cut is followed by an $\varepsilon-\eta$-good co-substantial cut. In this case we again conclude that the original cut is $\varepsilon-\eta$-good.

Lemma 14. Assume that $k>K_{1}$. If $S_{k}$ is a substantial cut, $S_{p_{0}}$ is a co-substantial cut that is $\varepsilon$ - $\eta$-good, where $p_{0}=Q(k)+1$, and $S_{q_{0}}<S_{Q(k)}$ with $q_{0}=Q\left(p_{0}\right)$, then the cut at $S_{k}$ is strongly $\varepsilon-\eta$-good.

Lemma 15. Assume that $k>K_{1}$. If $S_{k}$ is a co-substantial cut, $S_{p_{0}}$ is a co-substantial cut that is $\varepsilon$ - $\eta$-good, where $p_{0}=k+1$, and $S_{q_{0}}<S_{Q(k)}$ with $q_{0}=Q\left(p_{0}\right)$, then the cut at $S_{k}$ is strongly $\varepsilon-\eta$-good.

Again we give a proof of Lemma 14 and point out in some parenthetical remarks the differences of the proof of Lemma 15.

Proof. From the definition of $q_{0}$ we find that $q_{0}+1 \geq \min \{Q(k+1)$, $Q(Q(k)+1)\}$ and hence, since $k>K_{1}, S_{Q\left(q_{0}+1\right)}>20$. Thus, $S_{Q(k)}-S_{q_{0}} \geq$ $S_{q_{0}+1}-S_{q_{0}}=S_{Q\left(q_{0}+1\right)}>20$. Set $C=c_{S_{Q(k)}}$. (In the proof of Lemma 15 set $C=c_{S_{k}}$.) Put $h=|c-C|$. An argument similar to that for (10) gives

$$
h a^{S_{q_{0}}}\left(1-3 a^{-S_{q_{0}}}\right) \leq\left|c-c_{S_{p_{0}}}\right| \leq h a^{S_{q_{0}}} .
$$

Denote by $\phi$ the linear mapping that is the extension of $T_{a}^{S_{q_{0}}} \mid[c, C]$ onto $\mathbb{R}$. It is clear that the absolute value of the slope of $\phi$ is $a^{S_{q_{0}}}$.

As in the proof of Lemmas 12 and 13, without loss of generality assume that $z_{q_{0}}=\phi^{-1}(c) \in[c, C]$. Set $w=\phi^{-1}\left(y_{p_{0}}^{\prime}\right)$. Again, $\left|c-z_{q_{0}}\right|=a^{-S_{q_{0}}}\left|c-c_{S_{q_{0}}}\right|$ and $w$ is on the same side of $c$ as $\bar{y}_{k}$. (In the proof of Lemma 15, $w$ is on the same side of $c$ as $\bar{y}_{k}^{\prime}$.) The definition of $y_{p_{0}}^{\prime}$ and (14) give

$$
\left|c-y_{p_{0}}^{\prime}\right| \geq 4 h\left(1-3 a^{-S_{q_{0}}}\right) \geq 4 \cdot 0.99 h .
$$

Again in (15), $k>K_{1}$ and $q_{0} \geq \min \{Q(k+1), Q(Q(k)+1)\}$ imply that $S_{Q\left(q_{0}\right)} \geq 20$, i.e., $a^{-S_{Q\left(q_{0}\right)}}<0.001$. From $\left|w-z_{q_{0}}\right|=a^{-S_{q_{0}}}\left|c-y_{p_{0}}^{\prime}\right|$ and (15) we have

$$
\left|w-z_{q_{0}}\right| \geq 4 \cdot 0.99 a^{-S_{q_{0}}} h .
$$


Since $S_{p_{0}}$ is a co-substantial cut, we have $\left|c-c_{S_{q_{0}}}\right| \leq 3 h$. Hence,

$$
\left|c-z_{q_{0}}\right| \leq 3 h a^{-S_{q_{0}}} .
$$

Thus, from (16) and (17) we have

$$
|c-w| \geq 0.9 a^{-S_{q_{0}}} h=0.9 a^{\left(S_{Q(k)}-S_{q_{0}}\right)} a^{-S_{Q(k)}} h>8 a^{-S_{Q(k)}} h
$$

(remember that $\left.S_{Q(k)}-S_{q_{0}}>20\right)$.

Then $\left|c-\bar{y}_{k}\right|=8 a^{-S_{Q(k)}} h$ and (18) imply that $\bar{y}_{k} \in[c, w]$. (In the proof of Lemma 15, $\left|c-\bar{y}_{k}^{\prime}\right|=8 a^{-S_{Q(k)} h}$ and (18) imply that $\bar{y}_{k}^{\prime} \in[c, w]$.) Lastly, an argument similar to that in the proof of Lemmas 12 and 13 now implies that $S_{k}$ is strongly $\varepsilon-\eta$-good.

In the next proposition we put together the above four lemmas to obtain the induction property saying that if all "earlier" substantial and cosubstantial cuts are $\varepsilon-\eta$-good then so is the "next" one.

Proposition 3. Assume that $k>K_{1}$ and let $S_{k}$ be either a substantial or co-substantial cut. If all substantial or co-substantial cuts $S_{k^{\prime}}$ with $S_{Q\left(k^{\prime}\right)}<$ $S_{Q(k)}$ are $\varepsilon-\eta$-good, then $S_{k}$ is $\varepsilon-\eta$-good.

Proof. Assume that all substantial or co-substantial cuts $S_{k^{\prime}}$ with $S_{Q\left(k^{\prime}\right)}$ $<S_{Q(k)}$ are $\varepsilon-\eta$-good; when we refer to "the hypothesis" we mean precisely this assumption. Let $p_{0}$ and $q_{0}$ be as in Lemma 10 or 11, depending on whether $S_{k}$ is a substantial or a co-substantial cut.

CASE 1. Assume that $S_{p_{0}}$ is not a co-substantial cut. Then, from Lemma 10 or Lemma 11, $S_{q_{0}+l}$ is a substantial cut and $L=S_{q_{0}+l}=S_{q_{0}}+S_{Q\left(q_{0}+1\right)}+$ $\ldots+S_{Q\left(q_{0}+l\right)} \leq S_{Q(k)}$. Hence, $S_{Q\left(q_{0}+l\right)}<S_{Q(k)}$ and therefore (by hypothesis) $S_{q_{0}+l}$ is $\varepsilon-\eta$-good. Thus, by Lemmas 12 and $13, S_{k}$ is strongly $\varepsilon$ - $\eta$-good. Case 1 is complete.

If $S_{k}$ is a substantial cut, then (using (4)) $q_{0}=Q(Q(k)+1) \leq Q(k)$. Since $k>K_{1}$, we have $Q(k)>6$ and hence $\left|c_{S_{k}}-c_{S_{Q(k)}}\right|>8\left|c-c_{S_{k-1}}\right|$. If $S_{k}$ is a co-substantial cut, then $\left|c-c_{S_{Q(k)}}\right| \leq\left|c-c_{S_{k-1}}\right|$. Joining these two facts gives $\left|c-c_{S_{k}}\right| \geq\left|c-c_{S_{k-1}}\right|$. Thus, by $(3), q_{0}=Q(k+1) \leq Q(k)$. Therefore, whether $S_{k}$ is a substantial or co-substantial cut, we have

$$
S_{q_{0}} \leq S_{Q(k)} .
$$

Case 2. Assume that $S_{p_{0}}$ is a co-substantial cut and that $S_{q_{0}}<S_{Q(k)}$. Then, by hypothesis, $S_{p_{0}}$ is $\varepsilon$ - $\eta$-good, and therefore, by Lemmas 14 and 15 , $S_{k}$ is strongly $\varepsilon-\eta$-good. Case 2 is complete.

CASE 3. Assume that $S_{p_{0}}$ is a co-substantial cut and that $S_{q_{0}}=S_{Q(k)}$, i.e., $q_{0}=Q(k)$. For ease of notation, set $S_{q_{0}}=M$. 
If $S_{k}$ is a substantial cut, set $C=c_{S_{Q(k)}}$ and $h=h^{\prime}=\left|c-c_{S_{Q(k)} \mid}\right|$ If $S_{k}$ is a co-substantial cut, set $C=c_{S_{k}}, h=\left|c-c_{S_{k}}\right|$ and $h^{\prime}=\left|c-c_{S_{Q(k)}}\right|$. In both cases $h^{\prime} \leq h$ and, since $q_{0}=Q(k), c_{S_{p_{0}}}=T_{a}^{M}(C)$.

Without loss of generality, assume that $z_{q_{0}} \in[c, C]$. Denote by $\psi_{0}$ the linear mapping that is the extension of $T_{a}^{M} \mid[c, C]$ onto $\mathbb{R}$. Thus, $\psi_{0}(c)=c_{S_{q_{0}}}$ and $\psi_{0}([c, C])=\left[c_{S_{p_{0}}}, c_{S_{q_{0}}}\right]$. Clearly, $v_{0} \equiv z_{q_{0}}=\psi_{0}^{-1}(c)$ and

$$
\left|c-z_{q_{0}}\right|=a^{-M}\left|c-c_{S_{q_{0}}}\right|=a^{-M} h^{\prime} .
$$

Set $p_{1}=p_{0}+1$ and $q_{1}=Q\left(p_{1}\right)$. For $S_{k}$ a substantial cut, $S_{p_{0}}$ a co-substantial cut, and $q_{0}=Q(k)$ we obtain $\left|c-c_{S_{p_{0}}}\right|>\left|c-c_{S_{Q(k)}}\right|$ and hence (recall (3)) $q_{1}=Q\left(p_{0}+1\right) \leq Q(Q(k)+1)=q_{0}$. Also, for $S_{k}$ a co-substantial cut, $S_{p_{0}}$ a co-substantial cut, and $q_{0}=Q(k)$, we have $\left|c-c_{S_{p_{0}}}\right|>\left|c-c_{S_{k}}\right|$ and therefore $($ recall $(3)) q_{1}=Q\left(p_{0}+1\right) \leq Q(k+1)=Q\left(p_{0}\right)=q_{0}$. Either way, we have

$$
q_{1} \leq q_{0} .
$$

Assume that for $r \geq 1$ we have defined $p_{r}=p_{r-1}+1$ and $q_{r}=Q\left(p_{r}\right)$, and that $M \equiv S_{q_{0}}=S_{q_{1}}=\ldots=S_{q_{r}}$. Let $v_{r-1} \in[c, C]$ be the unique point such that $\psi_{r-1} \equiv T_{a}^{r M}$ maps $\left[v_{r-1}, C\right]$ linearly onto $\left[c, c_{S_{p_{r-1}}}\right]$. Note that the absolute value of the slope of $\psi_{r-1}$ is $a^{r M}$. Set $h_{r-1}=\left|c-c_{S_{p_{r-1}}}\right|$.

It follows from $M \equiv S_{q_{0}}=S_{q_{1}}=\ldots=S_{q_{r}}$ that $S_{p_{r}}$ is a co-substantial cut (in fact, $S_{p_{i}}$ is a co-substantial cut for $0 \leq i \leq r$ ).

Let $\psi_{r}$ denote the linear extension of $T_{a}^{M} \circ \psi_{r-1} \mid\left[v_{r-1}, C\right]$. Set $v_{r}=$ $\psi_{r}^{-1}(c)$. Then

$$
\left|v_{r}-v_{r-1}\right|=\left|\psi_{r}^{-1}(c)-\psi_{r}^{-1}\left(c_{S_{q_{r}}}=c_{S_{Q(k)}}\right)\right|=a^{-(r+1) M} h^{\prime} .
$$

Clearly, $\psi_{r}$ maps $\left[v_{r}, C\right]$ linearly onto $\left[c, c_{S_{p_{r}}}\right]$ and $\psi_{r}=T_{a}^{(r+1) M}$ on $\left[v_{r-1}, C\right]$. From (19), (20), $\left|c-v_{r-1}\right|=\left|c-z_{q_{0}}\right|\left(1+a^{-M}+a^{-2 M}+\ldots+a^{-(r-1) M}\right)$, and $\left|c-v_{r}\right|=\left|c-v_{r-1}\right|+\left|v_{r}-v_{r-1}\right|=\left|c-v_{r-1}\right|+a^{-(r+1) M} h^{\prime}$, we obtain

$$
\left|c-v_{r}\right| \leq \sum_{j=1}^{\infty} a^{-j M} h^{\prime}=\frac{a^{-M}}{1-a^{-M}} h^{\prime}<1.1 a^{-M} h .
$$

As previously done, for the numerical estimate of 1.1 in (21) we use the fact that $k>K_{1}$ and hence that $M=S_{q_{0}} \geq 20$; also recall that $h^{\prime} \leq h$.

Note that $h_{r} \equiv\left|\psi_{r}\left(\left[v_{r}, C\right]\right)\right|=\left|c-c_{S_{p_{r}}}\right|=a^{M} h_{r-1}-h^{\prime}$. If $h_{r} \geq \varepsilon$, then the cut at $S_{k}$ is $\varepsilon$ - $\eta$-good. To see this, set $J=\left[v_{r}, C\right]$. Then $T_{a}^{(r+1) M} \mid J$ is monotone and $\left|T_{a}^{(r+1) M}(J)\right|=h_{r} \geq \varepsilon$. We need $|J| / E_{J}>\eta$, where $E_{J}$ is the length of the shortest closed interval containing both $y_{k}$ and $J$ when $S_{k}$ is a substantial cut, or containing both $y_{k}^{\prime}$ and $J$ when $S_{k}$ is a co-substantial cut. We have $E_{J}=h+\left|c-y_{k}\right|$ if $S_{k}$ is a substantial cut and $E_{J}=h+\left|c-y_{k}^{\prime}\right|$ if $S_{k}$ is a co-substantial cut. Either way we have $E_{J}=h+4 a^{-M} h$. Next, from (21) we obtain $\left|c-v_{r}\right| \leq 0.002 h$. Hence, $|J|=h-\left|c-v_{r}\right| \geq 0.99 h$. 
Putting these together we have

$$
\frac{|J|}{E_{J}} \geq \frac{0.99 h}{h+4^{-M} h}>0.8 .
$$

Thus, $|J| / E_{J}>\eta$, since $\eta<1 / 2$.

Therefore, it remains to deal with the case where for some $r \geq 1$ we have $q_{i}=q_{0}$ for $1 \leq i \leq r, q_{r+1}<q_{0}$, and $h_{r}<\varepsilon$. In this case $S_{p_{r}}$ is still a co-substantial cut. Moreover, $\varepsilon_{1}>2 \varepsilon>2 h_{r}>\left|c_{S_{p_{r}}}-c_{S_{q_{r}}}\right|$ gives $p_{r}>K_{1}$ (by the definition of $\varepsilon_{1}$ ). Thus, applying either Lemma 13 or Lemma 15 we conclude that the co-substantial cut at $S_{p_{r}}$ is strongly $\varepsilon-\eta$-good.

More precisely, if $S_{p_{r+1}}$ is not a co-substantial cut, then we apply Lemma 13 with $k=p_{r}$ in the statement of the lemma. To do this we need the fact that $S_{q_{r}+l^{\prime \prime}}$ is $\varepsilon$ - $\eta$-good, where to avoid confusion we have replaced $l$ in Lemma 13 with $l^{\prime \prime}$. However, from Lemma 11, we have $q_{r+1}+l^{\prime \prime} \leq q_{r}=Q(k)$ and hence, by (4), $Q\left(q_{r+1}+l^{\prime \prime}\right)<Q(k)$. Thus, by hypothesis, $S_{q_{r+1}+l^{\prime \prime}}$ is $\varepsilon-\eta$-good.

Next, if $S_{p_{r+1}}$ is a co-substantial cut, then we apply Lemma 15 with $k=p_{r}$ in the statement of the lemma. To do this we need the fact that $q_{r+1}<Q(k)=q_{0}$ (which we have) and that $S_{p_{r+1}}$ is $\varepsilon-\eta$-good. But $q_{r+1}<$ $Q(k)$ and the hypothesis imply that $S_{p_{r+1}}$ is $\varepsilon$ - $\eta$-good. Thus, $S_{p_{r}}$ is strongly $\varepsilon-\eta$-good.

Let $\bar{y}_{p_{r}}^{\prime}$ be as in Definition 11, i.e., $\bar{y}_{p_{r}}^{\prime}$ is the point on the same side of $c$ as $c_{S_{q_{r}}}$ such that $\left|c-\bar{y}_{p_{r}}^{\prime}\right|=8 a^{-S_{q_{r}}}\left|c-c_{S_{p_{r}}}\right|=8 a^{-M} h_{r}$. Set $w=\psi_{r}^{-1}\left(\bar{y}_{p_{r}}^{\prime}\right)$. Then

$$
\left|v_{r}-w\right|=a^{-(r+1) M}\left|c-\bar{y}_{p_{r}}^{\prime}\right|=8 a^{-(r+2) M} h_{r} .
$$

Using (21) we get $\left|C-v_{r}\right|=h-\left|c-v_{r}\right| \geq h-1.1 h a^{-M}=h\left(1-1.1 a^{-M}\right)$. This and $h_{r}=a^{(r+1) M}\left|C-v_{r}\right|$ give $h_{r} \geq a^{(r+1) M} h\left(1-1.1 a^{-M}\right)>0.99 a^{(r+1) M} h$. Hence (also use (22)),

$$
\left|v_{r}-w\right| \geq 8 a^{-(r+2) M} \cdot 0.99 a^{(r+1) M} h>7 a^{-M} h .
$$

It follows from (21) and (23) that $w$ and $v_{r}$ lie on opposite sides of $c$. Hence, (again, use (21) and (23))

$$
|c-w|=\left|v_{r}-w\right|-\left|c-v_{r}\right|>5 a^{-M} h .
$$

Since the co-substantial cut at $S_{p_{r}}$ is strongly $\varepsilon$ - $\eta$-good, we may choose $I \subset\left[c, c_{S_{p_{r}}}\right]$ and $l \geq 1$ such that $T_{a}^{l} \mid I$ is monotone, $\left|T_{a}^{l}(I)\right|>\varepsilon$, and $|I|>\eta E_{I}$, where $E_{I}$ is the length of the shortest closed interval containing both $\bar{y}_{p_{r}}^{\prime}$ and $I$.

Set $J=\psi_{r}^{-1}(I)$. Then $J \subset\left[v_{r}, C\right]$ and denoting the length of the shortest closed interval containing both $w$ and $J$ by $E_{J}$ we have $|J|>\eta E_{J}$, by the linearity of $\psi_{r}^{-1}$. However, $\left|T_{a}^{(r+1) M+l}(J)\right|>\varepsilon$ and $T_{a}^{(r+1) M+l} \mid J$ is monotone, since $\psi_{r}\left|J=T_{a}^{(r+1) M}\right| J$. From (24) we have $|c-w|>4 a^{-M} h=4 a^{-M}|c-C|$. 
But $4 a^{-M}|c-C|$ equals $\left|c-y_{k}\right|$ when $S_{k}$ is a substantial cut, and equals $\left|c-y_{k}^{\prime}\right|$ when $S_{k}$ is a co-substantial cut. Therefore the cut at $S_{k}$ is $\varepsilon$ - $\eta$-good.

Definition 14. Fix $\delta<\min \left\{\left|c-c_{1}\right|,\left|c-c_{2}\right|\right\}$. For each $k \in \mathbb{N}$ such that $S_{k}$ is a substantial cut, set $A_{k, \delta}=\left\{I \subset\left[c, c_{S_{Q(k)}}\right] \mid\right.$ there exists $l \geq 1$ with $T_{a}^{l} \mid I$ monotone and $\left.\left|T_{a}^{l}(I)\right|>\delta\right\}$.

Definition 15. Fix $\delta<\min \left\{\left|c-c_{1}\right|,\left|c-c_{2}\right|\right\}$. For each $k \in \mathbb{N}$ such that $S_{k}$ is a co-substantial cut, set $B_{k, \delta}=\left\{I \subset\left[c, c_{S_{k}}\right] \mid\right.$ there exists $l \geq 1$ with $T_{a}^{l} \mid I$ monotone and $\left.\left|T_{a}^{l}(I)\right|>\delta\right\}$.

Remark. It follows from Proposition 2 that each of $A_{k, \delta}$ and $B_{k, \delta}$ is nonempty.

Lemma 16. Fix $\delta<\min \left\{\left|c-c_{1}\right|,\left|c-c_{2}\right|\right\}$ and suppose that $S_{k}$ is a substantial cut (resp. a co-substantial cut). Then there exists $0<\gamma<1$ such that for any $I \in A_{k, \delta}$ (resp. $I \in B_{k, \delta}$ ) we have $|I|<\gamma E_{I}$, where $E_{I}$ is the length of the shortest closed interval containing both $y_{k}$ (resp. $y_{k}^{\prime}$ ) and $I$.

Proof. We do the case when $S_{k}$ is a substantial cut, the case of a co-substantial cut being similar. Let $I$ and $E_{I}$ be as above. Set $g(x)=$ $x /\left(x+\left|c-y_{k}\right|\right)$ for $x \in[0,1]$. On $[0,1]$ the function $g(x)$ has a maximum when $x=1$; hence for $x \in[0,1]$, we have $g(x) \leq 1 /\left(1+\left|c-y_{k}\right|\right)$. This, $\left|c-y_{k}\right|+|I| \leq E_{I}$, and $|I| \leq 1$ give

$$
\frac{|I|}{E_{I}} \leq \frac{|I|}{|I|+\left|c-y_{k}\right|} \leq \frac{1}{1+\left|c-y_{k}\right|} .
$$

Thus,

$$
|I| \leq E_{I} \frac{1}{1+\left|c-y_{k}\right|} .
$$

The lemma now follows.

Definition 16. For each substantial cut $S_{k}$, set $\eta_{k}=\inf \{\gamma<1 \mid$ if $I \in A_{k, \varepsilon}$, then $\left.|I| \leq \gamma E_{I}\right\}$. Clearly, $\eta_{k}>0$ since $A_{k, \varepsilon} \neq \emptyset$.

Definition 17. For each co-substantial cut $S_{k}$, set $\beta_{k}=\inf \{\beta<1 \mid$ if $I \in B_{k, \varepsilon}$, then $\left.|I| \leq \beta E_{I}\right\}$. Again, $\beta_{k}>0$.

Theorem 2. Set $\eta^{*}=\inf \left\{\eta_{k} \mid S_{k}\right.$ is a substantial cut $\}$. Then $\eta^{*}>0$.

Proof. Set

$\alpha=\min \left\{\eta_{j} \mid j \leq K_{1}\right.$ and $S_{j}$ is a substantial cut $\}$,

$\beta=\min \left\{\beta_{j} \mid j \leq K_{1}\right.$ and $S_{j}$ is a co-substantial cut $\}$,

$\tau=\frac{1}{2} \min \left\{\alpha, \beta, \frac{1}{2}\right\}$,

$\mathcal{B}=\left\{k \mid S_{k}\right.$ is a substantial or a co-substantial cut that is $\varepsilon-\tau$-good $\}$. 
If either or both $\alpha, \beta$ are not defined (i.e., taking the minimum of an empty set), then delete them from the definition of $\tau$. We show that $\mathcal{B}=\emptyset$ and hence $\eta^{*} \geq \tau>0$, proving the theorem.

Suppose to the contrary that $\mathcal{B}$ is not empty. Then choose $k^{\prime} \in \mathcal{B}$ such that $Q\left(k^{\prime}\right) \leq Q(l)$ for all $l \in \mathcal{B}$. Since $k^{\prime} \in \mathcal{B}, S_{k^{\prime}}$ is either a substantial or a co-substantial cut that is not $\varepsilon-\tau$-good. First suppose that $k^{\prime}>K_{1}$. From the definition of $\mathcal{B}$, all substantial or co-substantial cuts $S_{m}$ with $Q(m)<Q\left(k^{\prime}\right)$ are $\varepsilon-\tau$-good and therefore, by Proposition $3, S_{k^{\prime}}$ is $\varepsilon$ - $\tau$-good, a contradiction. Thus, $k^{\prime} \leq K_{1}$. But then the definitions of $\alpha, \beta$, and $\tau$ imply that $S_{k^{\prime}}$ is also $\varepsilon$ - $\tau$-good, again a contradiction. Hence, $\mathcal{B}=\emptyset$.

COROLlary 1. There exists $\widetilde{\eta}>0$ such that every substantial cut $S_{k}$ is $\varepsilon-\widetilde{\eta}$-good.

Proposition 4. Let $\delta>0$. Then there exists a closed interval $J^{\prime} \subset$ $(a-\delta, a), k, l \in \mathbb{N}$, and $\eta_{1} \in(0,1)$ such that

(i) $J^{\prime} \subset \omega_{S_{k}}(a)$,

(ii) $\xi_{S_{k}+l^{\prime}} \mid J^{\prime}$ is monotone for $0<l^{\prime} \leq l$,

(iii) $\left|\xi_{S_{k}+l}\left(J^{\prime}\right)\right|>\varepsilon / 2$, and

(iv) $\left|J^{\prime}\right| / E_{J^{\prime}} \geq \eta_{1}$, where $E_{J^{\prime}}$ is the length of the shortest closed interval containing $J^{\prime}$ and $a$.

Moreover, $\eta_{1}$ is independent of $\delta$ (depends only on a and $\varepsilon$ ).

Proof. Let $\widetilde{\eta}$ be as in Corollary 1 and without loss of generality assume that $\widetilde{\eta}<1 / 10$. We write $c_{S_{k}}$ for $c_{S_{k}}(a)$ and $c_{S_{Q(k)}}$ for $c_{S_{Q(k)}}(a)$. For any $k$, one endpoint of $\xi_{S_{k}}\left(\omega_{S_{k}}^{\prime}(a)\right)$ is $c_{S_{k}}$ and we denote the other endpoint by $c_{S_{k}}^{\prime}$; here we take $\omega_{S_{k}}^{\prime}(a)$ to be closed. In Figure $1, c_{S_{k}}^{\prime}=\xi_{S_{k}}(u)$. Choose $k \in \mathbb{N}$ such that $S_{k}$ is a substantial cut, $\omega_{S_{k}}^{\prime}(a) \subset(a-\delta, a], k>K_{0}$ from Lemma 2, $\left(1+\varepsilon_{0}\right) a^{-S_{k}} /(\alpha \widetilde{\eta}(a-1))<\min \{0.1, \varepsilon / 8\}$ (here $\alpha$ is from Lemma 3), and

$$
\frac{\left|c_{S_{k}}^{\prime}-c_{S_{Q(k)}}\right|}{\left|c_{S_{k}}-c_{S_{k}}^{\prime}\right|}<\frac{\tilde{\eta}}{1000} .
$$

For (25) use Lemma 7. From the definition of $\widetilde{\eta}, S_{k}$ is $\varepsilon-\widetilde{\eta}$-good and hence choose $I \subset\left[c, c_{S_{Q(k)}}\right]$ and $l \geq 1$ such that $T_{a}^{l} \mid I$ is monotone, $\left|T_{a}^{l}(I)\right|>\varepsilon$, and

$$
|I| \geq \widetilde{\eta} E_{I}
$$

where $E_{I}$ is the length of the shortest interval containing both $y_{k}$ and $I$. Let $I^{\prime} \subset I$ be concentric with $I$ and such that $\left|I^{\prime}\right|=0.8|I|$. From (25) and (26) it follows that $I^{\prime} \subset \xi_{S_{k}}\left(\omega_{S_{k}}^{\prime}(a)\right)=\left[c_{S_{k}}, c_{S_{k}}^{\prime}\right]$. In what follows we will write $\xi_{S_{k}}^{-1}$ for the well defined (since $\xi_{S_{k}}$ is strictly monotone on $\omega_{S_{k}}^{\prime}(a)$ ) inverse of $\xi_{S_{k}}$ restricted to $\omega_{S_{k}}^{\prime}(a)$. Set $J^{\prime}=\xi_{S_{k}}^{-1}\left(I^{\prime}\right)$. Since $\omega_{S_{k}}^{\prime}(a) \subset(a-\delta, a]$, we obtain (i), i.e. $J^{\prime} \subset \omega_{S_{k}}(a)$. 
Let $D_{I^{\prime}}$ be the smallest closed interval containing $c_{S_{k}}$ and $I^{\prime}$; denote the length of $D_{I^{\prime}}$ by $E_{I^{\prime}}$. It is not difficult to see that $D_{I^{\prime}} \subset \xi_{S_{k}}\left(\omega_{S_{k}}^{\prime}(a)\right)$. Notice that

$$
E_{I^{\prime}}<E_{I} \leq|I| / \widetilde{\eta}
$$
have

For $x \in \xi_{S_{k}}\left(\omega_{S_{k}}^{\prime}(a)\right)=\left[c_{S_{k}}, c_{S_{k}}^{\prime}\right]$ set $e(x)=\xi_{S_{k}}^{-1}(x)$. From Lemma 2 we

$$
\frac{1}{1+\varepsilon_{0}}\left|\xi_{S_{k}}^{\prime}(a)\right| \leq\left|\xi_{S_{k}}^{\prime}(e(x))\right| \leq\left(1+\varepsilon_{0}\right)\left|\xi_{S_{k}}^{\prime}(a)\right|
$$

for any $x \in \xi_{S_{k}}\left(\omega_{S_{k}}^{\prime}(a)\right)$. Similarly we have,

$$
\frac{1}{1+\varepsilon_{0}}\left|e^{\prime}\left(c_{S_{k}}\right)\right| \leq\left|e^{\prime}(x)\right| \leq\left(1+\varepsilon_{0}\right)\left|e^{\prime}\left(c_{S_{k}}\right)\right|
$$

for any $x \in \xi_{S_{k}}\left(\omega_{S_{k}}^{\prime}(a)\right)$. From Lemma 3 and (28) we have

$$
\frac{1+\varepsilon_{0}}{\alpha} a^{-S_{k}} \geq\left|e^{\prime}(x)\right| \geq \frac{1}{\beta\left(1+\varepsilon_{0}\right)} a^{-S_{k}}
$$

for any $x \in \xi_{S_{k}}\left(\omega_{S_{k}}^{\prime}(a)\right) \supset D_{I^{\prime}}$.

Assume that $a^{\prime} \in \xi_{S_{k}}^{-1}\left(D_{I^{\prime}}\right)$ and let $x^{\prime}=\xi_{S_{k}}\left(a^{\prime}\right)$. Then, from (29),

$$
\left|a-a^{\prime}\right|=\left|e\left(c_{S_{k}}\right)-e\left(x^{\prime}\right)\right| \leq \int_{D_{I^{\prime}}}\left|e^{\prime}(x)\right| d x \leq \frac{1+\varepsilon_{0}}{\alpha} a^{-S_{k}} E_{I^{\prime}} .
$$

It follows from (30) and Lemma 6 that

$$
\begin{aligned}
\left|T_{e(x)}^{l^{\prime}}(x)-T_{a}^{l^{\prime}}(x)\right| & \leq|a-e(x)| \frac{a^{l^{\prime}}-1}{a-1}<|a-e(x)| \frac{a^{l^{\prime}}}{a-1} \\
& <\frac{1+\varepsilon_{0}}{\alpha} a^{-S_{k}} E_{I^{\prime}} \frac{a^{l^{\prime}}}{a-1}
\end{aligned}
$$

for any $x \in D_{I^{\prime}}$ and $0<l^{\prime} \leq l$.

Claim 1. For $0<l^{\prime} \leq l, \xi_{S_{k}+l^{\prime}} \mid J^{\prime}$ is monotone, i.e., (ii) holds.

Proof. Suppose to the contrary that there exists some $x \in I^{\prime}=\xi_{S_{k}}\left(J^{\prime}\right)$ such that $\xi_{S_{k}+l^{\prime}}(e(x))=T_{e(x)}^{l^{\prime}}(x)=c$. Then from (27) and (31),

$$
\begin{aligned}
\left|T_{a}^{l^{\prime}}(x)-c\right| & =\left|T_{a}^{l^{\prime}}(x)-T_{e(x)}^{l^{\prime}}(x)\right|<\frac{1+\varepsilon_{0}}{\alpha} a^{-S_{k}} E_{I^{\prime}} \frac{a^{l^{\prime}}}{a-1} \\
& <\frac{1+\varepsilon_{0}}{\alpha} a^{-S_{k}} \frac{|I|}{\widetilde{\eta}} \cdot \frac{a^{l^{\prime}}}{a-1}<0.1|I| a^{l^{\prime}} .
\end{aligned}
$$

Since $x \in I^{\prime}$, it follows that $[x-0.1|I|, x+0.1|I|] \subset I$. Next, since $T_{a}^{l^{\prime}} \mid I$ is monotone, we obtain

$$
\left[T_{a}^{l^{\prime}}(x)-0.1 a^{l^{\prime}}|I|, T_{a}^{l^{\prime}}(x)+0.1 a^{l^{\prime}}|I|\right] \subset T_{a}^{l^{\prime}}(I)
$$


for $0<l^{\prime} \leq l$. Now, (32) and (33) imply that $c \in T_{a}^{l^{\prime}}(I)$. However, by assumption, $T_{a}^{l^{\prime}}$ is monotone on $I$ for $0<l^{\prime} \leq l$ and hence $c \notin T_{a}^{l^{\prime}}(I)$. This contradiction completes the proof of Claim 1.

Claim 2. We have $\left|\xi_{S_{k}+l}\left(J^{\prime}\right)\right|>\varepsilon / 2$, i.e., (iii) holds.

Proof. It follows from Claim 1 that $\xi_{S_{k}+l^{\prime}} \mid J^{\prime}$ is monotone for all $0<$ $l^{\prime} \leq l$ and hence, in particular, $\xi_{S_{k}+l} \mid J^{\prime}$ is monotone. Choose $a_{1}$ and $a_{2}$ such that $J^{\prime}=\left[a_{1}, a_{2}\right]$. Then, $\left[\xi_{S_{k}}\left(a_{1}\right), \xi_{S_{k}}\left(a_{2}\right)\right]=I^{\prime}$ and

$$
\left|T_{a}^{l}\left(\xi_{S_{k}}\left(a_{1}\right)\right)-T_{a}^{l}\left(\xi_{S_{k}}\left(a_{2}\right)\right)\right|=a^{l}\left|I^{\prime}\right|=a^{l} \frac{\left|I^{\prime}\right|}{|I|}|I|=\frac{\left|I^{\prime}\right|}{|I|}\left|T_{a}^{l}(I)\right| \geq \frac{8}{10} \varepsilon .
$$

For $i=1,2$ (recall $(27)$ and (31)),

$$
\begin{aligned}
\left|T_{a}^{l}\left(\xi_{S_{k}}\left(a_{i}\right)\right)-\xi_{S_{k}+l}\left(a_{i}\right)\right| & =\left|T_{a}^{l}\left(\xi_{S_{k}}\left(a_{i}\right)\right)-T_{a_{i}}^{l}\left(\xi_{S_{k}}\left(a_{i}\right)\right)\right| \\
& <\frac{1+\varepsilon_{0}}{\alpha} a^{-S_{k}} E_{I^{\prime}} \frac{a^{l}}{a-1} \\
& \leq \frac{1+\varepsilon_{0}}{\alpha} a^{-S_{k}} \frac{|I|}{\widetilde{\eta}} \cdot \frac{a^{l}}{a-1} \\
& =\frac{1+\varepsilon_{0}}{\alpha} a^{-S_{k}} \frac{1}{\widetilde{\eta}} \cdot \frac{1}{a-1}\left|T_{a}^{l}(I)\right| \\
& \leq \frac{1+\varepsilon_{0}}{\alpha} a^{-S_{k}} \frac{1}{\widetilde{\eta}} \cdot \frac{1}{a-1}<\frac{\varepsilon}{8} .
\end{aligned}
$$

Lastly, (34) and (35) imply that $\left|\xi_{S_{k}+l}\left(J^{\prime}\right)\right|=\left|\xi_{S_{k}+l}\left(a_{1}\right)-\xi_{S_{k}+l}\left(a_{2}\right)\right|>\varepsilon / 2$. Claim 2 is thus proved.

Again, $\left|I^{\prime}\right|=0.8|I| \geq 0.8 \widetilde{\eta} E_{I}>0.8 \widetilde{\eta} E_{I^{\prime}}$ and hence (recall $(29)$ )

$$
\left|J^{\prime}\right|=\int_{I^{\prime}}\left|e^{\prime}(x)\right| d x \geq \frac{1}{\beta\left(1+\varepsilon_{0}\right)} a^{-S_{k}}\left|I^{\prime}\right|>\frac{1}{\beta\left(1+\varepsilon_{0}\right)} a^{-S_{k}} \cdot 0.8 \widetilde{\eta} E_{I^{\prime}}
$$

Letting $D_{J^{\prime}}$ denote the shortest interval containing $a$ and $J^{\prime}$ and setting $E_{J^{\prime}}=\left|D_{J^{\prime}}\right|$ we have (recall (29))

$$
E_{J^{\prime}} \leq \int_{D_{I^{\prime}}}\left|e^{\prime}(x)\right| d x \leq \frac{1+\varepsilon_{0}}{\alpha} a^{-S_{k}} E_{I^{\prime}}
$$

Joining (36) and (37) we obtain

$$
\frac{\left|J^{\prime}\right|}{E_{J^{\prime}}}>\frac{0.8 \widetilde{\eta} \alpha}{\beta\left(1+\varepsilon_{0}\right)^{2}} \equiv \eta_{1}
$$

Thus, (iv) holds.

7. Proof of Theorem 1. We have already shown that $\mathcal{P}$ and $\mathcal{H}=\{a \in$ $[\sqrt{2}, 2] \mid \liminf _{k \rightarrow \infty} Q_{a}(k)<\infty$ and $\left.a \notin \mathcal{D} \cup \mathcal{P}\right\}$ are $\sigma$-porous. Hence, to prove Theorem 1, it remains to show that $\mathcal{I}$ is $\sigma$-porous. 
For each pair $r_{1}<r_{2} \in \mathbb{Q}$ set

$$
\begin{aligned}
\mathcal{I}_{r_{1}, r_{2}}=\left\{a \mid\left(r_{1}, r_{2}\right) \subset\left[\xi_{2}(a), \xi_{1}(a)\right], \xi_{n}(a) \notin\right. & \left(r_{1}, r_{2}\right) \text { for all } n, \\
& \text { and } \left.\lim _{k \rightarrow \infty} Q_{a}(k)=\infty\right\} .
\end{aligned}
$$

We will show that each nonempty $\mathcal{I}_{r_{1}, r_{2}}$ is porous and therefore that $\mathcal{I}$ is $\sigma$-porous. Theorem 1 will then immediately follow.

Fix $r_{1}<r_{2} \in \mathbb{Q}$ such that $\mathcal{I}_{r_{1}, r_{2}} \neq \emptyset$ and fix $a \in \mathcal{I}_{r_{1}, r_{2}}$. Let $\varepsilon>0$ be as defined in Section 6 for the fixed $a$. Let $\left\{\delta_{i}>0\right\}$ be such that $\lim _{i \rightarrow \infty} \delta_{i}=0$. For each $i$ apply Proposition 4 with the fixed $a, \varepsilon$, and $\delta=\delta_{i}$, to generate sets $J_{i}^{\prime}$ and positive integers $k_{i}$ and $l_{i}$ which satisfy for each $i$ the conditions (i)-(iv) of Proposition 4. Since $\eta_{1}$ from Proposition 4 is independent of the choice of $\delta$ (depends only on $a$ and $\varepsilon$ ) we have a common $\eta_{1}$ for all $i$.

Let $\mathcal{P}$ be a partition of $[0,1]$ into subintervals of length $\varepsilon / 4$, with perhaps the partition element containing 0 having a smaller length. Set $r_{1}^{\prime}=r_{1}+$ $\left(r_{2}-r_{1}\right) / 4$ and $r_{2}^{\prime}=r_{2}-\left(r_{2}-r_{1}\right) / 4$. Since $T_{a}$ is leo, for each $I \in \mathcal{P}$ there exists a closed interval $U_{I} \subset I$ and $k_{I} \in \mathbb{N}$ such that $T_{a}^{k_{I}}\left(U_{I}\right) \subset\left(r_{1}^{\prime}, r_{2}^{\prime}\right)$. Let $\gamma>0$ be such that if $a^{\prime} \in(a-\gamma, a)$. Then for each $I \in \mathcal{P}$ we have $T_{a^{\prime}}^{k_{I}}\left(U_{I}\right) \subset\left(r_{1}, r_{2}\right)$. Passing to a subsequence if needed, assume that

$$
J_{i}^{\prime} \subset(a-\gamma, a)
$$

for all $i$.

Since $\mathcal{P}$ is a finite partition, $\left|\xi_{S_{k_{i}}+l_{i}}\left(J_{i}^{\prime}\right)\right|>\varepsilon / 2$ for all $i$, and $|I| \leq \varepsilon / 4$ for all $I \in \mathcal{P}$, we may choose $I^{*} \in \mathcal{P}$ such that $I^{*} \subset \xi_{S_{k_{i}}+l_{i}}\left(J_{i}^{\prime}\right)$ for infinitely many $i$. Again, passing to a subsequence if needed, assume that $I^{*} \subset \xi_{S_{k_{i}}+l_{i}}\left(J_{i}^{\prime}\right)$ for all $i$. Next for each $i$ set

$$
J_{i}^{*}=\xi_{S_{k_{i}}+l_{i}}^{-1}\left(U_{I^{*}}\right) .
$$

Then for all $i, J_{i}^{*} \subset J_{i}^{\prime}$ and

$$
\xi_{S_{k_{i}}+l_{i}+k_{I^{*}}}\left(J_{i}^{*}\right)=\xi_{k_{I^{*}}}\left(U_{I^{*}}\right) .
$$

It follows from (39) and (40) that for all $i, \xi_{S_{k_{i}}+l_{i}+k_{I^{*}}}\left(J_{i}^{*}\right) \subset\left(r_{1}, r_{2}\right)$. Hence,

$$
J_{i}^{*} \not \subset \mathcal{I}_{r_{1}, r_{2}}
$$

for all $i$.

Claim 1. For each $i$,

$$
\left|J_{i}^{\prime}\right| \leq \frac{1+\varepsilon_{0}}{\left|U_{I^{*}}\right|}\left|J_{i}^{*}\right| .
$$

Proof. For each $i$ set $H_{i}=\xi_{S_{k_{i}}+l_{i}}\left(J_{i}^{\prime}\right)$. Set $M=\max _{x \in J_{i}^{\prime}}\left\{\left|\xi_{S_{k_{i}}+l_{i}}^{\prime}(x)\right|\right\}$ and $m=\min _{x \in J_{i}^{\prime}}\left\{\left|\xi_{S_{k_{i}}+l_{i}}^{\prime}(x)\right|\right\}$. Then

$$
\left|U_{I^{*}}\right|=\int_{J_{i}^{*}}\left|\xi_{S_{k_{i}}+l_{i}}^{\prime}(x)\right| d x \leq M\left|J_{i}^{*}\right|,
$$




$$
\left|H_{i}\right|=\int_{J_{i}^{\prime}}\left|\xi_{S_{k_{i}}+l_{i}}^{\prime}(x)\right| d x \geq m\left|J_{i}^{\prime}\right| .
$$

Combining (42) and (43) and using Lemma 2 we have

$$
\frac{\left|U_{I^{*}}\right|}{\left|H_{i}\right|} \leq \frac{M\left|J_{i}^{*}\right|}{m\left|J_{i}^{\prime}\right|} \leq\left(1+\varepsilon_{0}\right) \frac{\left|J_{i}^{*}\right|}{\left|J_{i}^{\prime}\right|} .
$$

Hence,

$$
\frac{\left|J_{i}^{*}\right|}{\left|J_{i}^{\prime}\right|} \geq \frac{1}{1+\varepsilon_{0}} \cdot \frac{\left|U_{I^{*}}\right|}{\left|H_{i}\right|} \geq \frac{\left|U_{I^{*}}\right|}{1+\varepsilon_{0}} ;
$$

use $\left|H_{i}\right| \leq 1$. This shows Claim 1 .

For each $i$ let $\Delta_{i}$ be the length of the smallest closed interval containing $a$ and $J_{i}^{*}$. We need to find $\lambda>0$ such that

$$
\left|J_{i}^{*}\right| / \Delta_{i} \geq \lambda
$$

for all $i$. Write

$$
\frac{\left|J_{i}^{*}\right|}{\Delta_{i}}=\frac{\left|J_{i}^{*}\right|}{\left|J_{i}^{\prime}\right|} \cdot \frac{\left|J_{i}^{\prime}\right|}{\Delta_{i}} .
$$

From Proposition 4 we have $\left|J_{i}^{\prime}\right| / E_{J_{i}^{\prime}} \geq \eta_{1}$ for each $i$, and by definition $\Delta_{i} \leq E_{J_{i}^{\prime}}$ for all $i$; therefore

$$
\left|J_{i}^{\prime}\right| / \Delta_{i} \geq \eta_{1}
$$

for all $i$. Joining (44) and (45), we see that to get a lower bound for $\left|J_{i}^{*}\right| / \Delta_{i}$ it suffices to have a lower bound for $\left|J_{i}^{*}\right| /\left|J_{i}^{\prime}\right|$; but this is precisely Claim 1. Thus Claim 1, (44), and (45) give

$$
\frac{\left|J_{i}^{*}\right|}{\Delta_{i}} \geq \frac{\left|U_{I^{*}}\right|}{1+\varepsilon_{0}} \eta_{1} \equiv \lambda
$$

for all $i$; note that $\lambda$ is independent of $i$. Recalling (41) we now have, for each $i$

$$
\left|J_{i}^{*}\right| / \Delta_{i} \geq \lambda \quad \text { and } \quad J_{i}^{*} \cap \mathcal{I}_{r_{1}, r_{2}}=\emptyset .
$$

Thus $\mathcal{I}_{r_{1}, r_{2}}$ is porous.

\section{References}

[1] L. Block and W. A. Coppel, Dynamics in One Dimension, Lecture Notes in Math. 1513, Springer, New York, 1992.

[2] K. Brucks, B. Diamond, M. V. Otero-Espinar and C. Tresser, Dense orbits of critical points for the tent map, in: Contemp. Math. 117, Amer. Math. Soc., 1991, 57-61.

[3] K. Brucks and M. Misiurewicz, The trajectory of the turning point is dense for almost all tent maps, Ergodic Theory Dynam. Systems 16 (1996), 1173-1183. 
[4] H. Bruin, Invariant measures of interval maps, Ph.D. thesis, Delft, 1994.

[5] H. Bruin, Combinatorics of the kneading map, Internat. J. Bifur. Chaos Appl. Sci. Engrg. 5 (1995), 1339-1349.

[6] -, Quasi-symmetry of conjugacies between interval maps, Nonlinearity 9 (1996), 1191-1207.

[7] - , Topological conditions for the existence of absorbing Cantor sets, Trans. Amer. Math. Soc. 350 (1998), 2229-2263.

[8] - For almost every tent map, the turning point is typical, Fund. Math. 155 (1998), 215-235.

[9] F. Hofbauer, The topological entropy of the transformation $x \mapsto a x(1-x)$, Monatsh. Math. 90 (1980), 117-141.

[10] F. Hofbauer and G. Keller, Quadratic maps without asymptotic measure, Comm. Math. Phys. 127 (1990), 319-337.

[11] W. de Melo and S. van Strien, One-Dimensional Dynamics, Springer, New York, 1993.

[12] D. Preiss and L. Zajíček, Fréchet differentiation of convex functions in a Banach space with a separable dual, Proc. Amer. Math. Soc. 91 (1984), 202-204.

[13] D. L. Renfro, On some various porosity notions, preprint, 1995.

[14] D. Sands, Topological conditions for positive Lyapunov exponent in unimodal maps, Ph.D. thesis, Cambridge, 1994.

[15] S. van Strien, Smooth dynamics on the interval, in: New Directions in Dynamical Systems, London Math. Soc. Lecture Note Ser. 127, Cambridge Univ. Press, Cambridge, 1988, 57-119.

[16] B. S. Thomson, Real Functions, Lecture Notes in Math. 1170, Springer, New York, 1985.

[17] L. Zajíček, Porosity and $\sigma$-porosity, Real Anal. Exchange 13 (1987-88), 314-347.

Department of Mathematical Sciences University of Wisconsin at Milwaukee Milwaukee, WI 53201, U.S.A.

E-mail: kmbrucks@csd.uwm.edu
Department of Analysis Eötvös Loránd University Múzeum krt. 6-8 H-1088 Budapest, Hungary E-mail: buczo@cs.elte.hu 